\title{
The Stripe 82 Massive Galaxy Project. III. A Lack of Growth among Massive Galaxies
}

\author{
Kevin Bundy ${ }^{1,2}$ (1) , Alexie Leauthaud ${ }^{2}$ (1) , Shun Saito $^{3}$, Claudia Maraston ${ }^{4}$, David A. Wake ${ }^{5}$ (1), and Daniel Thomas ${ }^{4}$ \\ ${ }^{1}$ UC Observatories, MS: UCO Lick, UC Santa Cruz, 1156 High St., Santa Cruz, CA 95064, USA \\ ${ }^{2}$ Department of Astronomy \& Astrophysics, UC Santa Cruz, 1156 High St., Santa Cruz, CA 95064, USA \\ ${ }^{3}$ Max-Planck-Institut für Astrophysik, Karl-Schwarzschild-Str. 1, D-85741 Garching, Germany \\ ${ }^{4}$ Institute of Cosmology and Gravitation, University of Portsmouth, Portsmouth, UK \\ ${ }^{5}$ Department of Physical Sciences,The Open University, Milton Keynes, MK7 6AA, UK \\ Received 2017 August 4; revised 2017 November 1; accepted 2017 November 2; published 2017 December 8
}

\begin{abstract}
The average stellar mass $\left(M_{*}\right)$ of high-mass galaxies $\left(\log M_{*} / M_{\odot}>11.5\right)$ is expected to grow by $\sim 30 \%$ since $z \sim 1$, largely through ongoing mergers that are also invoked to explain the observed increase in galaxy sizes. Direct evidence for the corresponding growth in stellar mass has been elusive, however, in part because the volumes sampled by previous redshift surveys have been too small to yield reliable statistics. In this work, we make use of the Stripe 82 Massive Galaxy Catalog (S82-MGC) to build a mass-limited sample of 41,770 galaxies $\left(\log M_{*} / M_{\odot}>11.2\right)$ with optical-to-near-IR photometry and a large fraction $(>55 \%)$ of spectroscopic redshifts. Our sample spans $139 \mathrm{deg}^{2}$, significantly larger than most previous efforts. After accounting for a number of potential systematic errors, including the effects of $M_{*}$ scatter, we measure galaxy stellar mass functions over $0.3<z<0.65$ and detect no growth in the typical $M_{*}$ of massive galaxies with an uncertainty of $9 \%$. This confidence level is dominated by uncertainties in the star formation (SF) history assumed for $M_{*}$ estimates, although our inability to characterize low-surface-brightness outskirts may be the most important limitation of our study. Even among these high-mass galaxies, we find evidence for differential evolution when splitting the sample by recent SF activity. While low-SF systems appear to become completely passive, we find a mostly subdominant population of galaxies with residual, but low rates of $\mathrm{SF}\left(\sim 1 M_{\odot} \mathrm{yr}^{-1}\right)$ whose number density does not evolve. Interestingly, these galaxies become more prominent at higher $M_{*}$, representing $\sim 10 \%$ of all galaxies at $10^{12} M_{\odot}$ and perhaps dominating at even larger masses.
\end{abstract}

Key words: galaxies: abundances

\section{Introduction}

Hierarchical growth, by which increasingly larger structures are built through the assembly of smaller ones, is a major feature of the $\Lambda \mathrm{CDM}$ paradigm. Its imprint on the evolving abundance of galaxy clusters is an important cosmological probe (e.g., Vikhlinin et al. 2009), and evidence for hierarchical growth has also been reported among group-scale halos (Williams et al. 2012). Because galaxies reside in dark matter halos, one also expects patterns of hierarchical growth in observables that trace galaxy mass, such as stellar mass, $M_{*}$ (Stringer et al. 2009), or assembly history (e.g., Gu et al. 2016), including morphology (Wilman et al. 2013) and size (Zhao et al. 2015).

Indeed, recent galaxy formation models employing both hydrodynamic simulations and semi-analytic recipes predict a galaxy stellar mass function that grows substantially at the high-mass end, tracking to some degree the dark matter halo mass function (e.g., de Lucia \& Blaizot 2007; Guo et al. 2011; Furlong et al. 2015; Torrey et al. 2017). While various but still uncertain mechanisms limit star formation (SF) among both low- and high-mass galaxies (Benson et al. 2003), thus working to decouple $M_{*}$ from $M_{\text {halo }}$, late-time growth in $M_{*}$ among the most massive galaxies (with no ongoing SF) is still expected as a result of galaxy mergers (e.g., Lee \& Yi 2013; Qu et al. 2017).

The role of such mergers in driving high-mass galaxy growth at $z \lesssim 2$ has been the subject of recent observational work (e.g., Bundy et al. 2009; Lotz et al. 2011; Casteels et al. 2014; Mundy et al. 2017) and the basis of theoretical explanations for how massive compact spheroidals at $z \approx 2$ grow significantly in size by the present day (e.g., Hopkins et al. 2010; Nipoti et al. 2012; Hilz et al. 2013; Welker et al. 2017). Comparisons of the predicted growth in diffuse outer components required to drive increasing size estimates appear to be consistent with observed (minor) merger rates, at least for $z \lesssim 1$ (LópezSanjuan et al. 2012; Newman et al. 2012; Ownsworth et al. 2014).

The rate of merging required to grow high-mass galaxies sufficiently in size should also add significantly to their stellar mass (e.g., Lidman et al. 2013). An implied $~ 30 \%$ growth in $M_{*}$ since $z \sim 1$ is typical and should be reflected in derived $M_{*}$ growth rates from evolving galaxy stellar mass functions. Recent observational results, however, have largely indicated little or no evolution in the total mass function and a lack of $M_{*}$ growth from $z \sim 1$ to today (e.g., Brammer et al. 2011; Davidzon et al. 2013; Ilbert et al. 2013; Moustakas et al. 2013; Muzzin et al. 2013). How can hierarchical assembly explain the growth in galaxy sizes but not simultaneously yield growth in galaxy masses?

One answer is that we are only beginning to survey the large volumes required to detect the expected signal. Stringer et al. (2009) argued that tens, if not hundreds, of $\mathrm{deg}^{2}$ are required to statistically confirm hierarchical growth in galaxy mass functions. In this regime, attention to systematic uncertainties is critical (e.g., Marchesini et al. 2009). Much recent work on galaxy number densities has prioritized reaching higher redshifts with "pencil-beam" surveys that sample combined areas of only a few $\operatorname{deg}^{2}$. Moustakas et al. (2013), which is based on the $5.5 \mathrm{deg}^{2}$ PRIsm MUlti-object Survey (PRIMUS; Coil et al. 2011) and Davidzon et al. (2013), which analyzes 
early data obtained over $10.3 \mathrm{deg}^{2}$ from the VIMOS Public Extragalactic Redshift Survey (VIPERS; Guzzo et al. 2014), represent early attempts to extend $M_{*}$-complete redshift surveys to larger areas.

To reach larger cosmic volumes, the challenge of building complete spectroscopic samples makes photometric redshifts (photo-zs) attractive, especially as wide- and deep-imaging surveys become more prevalent. Moutard et al. (2016a) exploited VIPERS PDR-1 (Garilli et al. 2014) spectroscopic redshifts (spec-zs), Canada-France-Hawaii Telescope (CFHT), and GALEX photometry obtained over the VIPERS footprint to construct a photo-z-based galaxy sample with $0.2<z<1.5$ that is complete to $\approx 10^{10} M_{\odot}$ at $z=1$. This sample is used to study the evolving mass function over $22 \mathrm{deg}^{2}$ in Moutard et al. (2016b). Some years earlier, Matsuoka \& Kawara (2010) combined and reanalyzed imaging data from the Sloan Digital Sky Survey (SDSS) Stripe 82 Coadd (see Annis et al. 2014) and the UK Infrared Deep Sky Survey Large Area Survey (UKIDSSLAS; Lawrence et al. 2007) in order to derive photometric redshifts and study galaxy mass functions over $55 \mathrm{deg}^{2}$ at $z<1$. The analysis we present in this work utilizes these same data sets, which have become more complete since Matsuoka \& Kawara (2010) and can be combined with a substantial number of spec-zs to yield an $M_{*}$-complete sample comprising $139 \mathrm{deg}^{2}$ with $z<0.7$, part of which we term the Stripe 82 Massive Galaxy Catalog (S82-MGC; Bundy et al. 2015).

With tens of square degrees surveyed, both Matsuoka \& Kawara (2010) and Moutard et al. (2016a) claimed to detect growth in the number density of the most massive galaxies, although the amplitude of the detected evolution is inconsistent. At $\log M_{*} / M_{\odot}>11.5$, Matsuoka \& Kawara (2010) found nearly an order of magnitude increase in number density from $z \sim 1$ to $z \sim 0.3$, while Moutard et al. (2016a) measured only a factor of 2 increase. Meanwhile, initial work by Capozzi et al. (2017) exploits $155 \mathrm{deg}^{2}$ of the Dark Energy Survey (DES) Science Verification Data to report a modest decrease in $M_{*}$ at the highest masses since $z \approx 1$.

This discrepancy highlights the challenge of this measurement and raises concerns about uncertain (perhaps catastrophically uncertain) photo-zs, as well as possibly larger-than-expected contributions from "cosmic variance." Both issues can be addressed by turning to very wide spec- $z$ surveys designed to constrain cosmological parameters via angular clustering. The drawback of these surveys, which can span thousands of $\mathrm{deg}^{2}$, is the difficulty in accounting for incompleteness owing to the selection criteria. Relevant here is early work by Wake et al. (2006) that detected no evolution in the number density of "luminous red galaxies" as measured at $z \sim 0.55$ by the $2 \mathrm{dF}$ SDSS LRG and QSO (2SLAQ) survey and at $z \sim 0.2$ by the SDSS. Finding a luminosity function consistent with that measured in the magnitude-limited COMBO17 survey, Wake et al. (2006) argued that the no-evolution conclusion applies broadly to the high-mass galaxy population.

Maraston et al. (2013) presented a more recent example of this approach, using spec-zs from the SDSS-III Baryon Oscillation Spectroscopic Survey (BOSS; Dawson et al. 2013) taken from the $0.43<z<0.7$ "constant mass" (CMASS) sample. Instead of correcting for incompleteness in the CMASS sample, Maraston et al. (2013) applied the same CMASS selection cuts to simulated data from a semi-analytic model. Doing so indicated that at least for $z \lesssim 0.6$, CMASS reaches high completeness $(>90 \%)$ at the highest masses (a conclusion that is confirmed and quantified in Leauthaud et al. 2016). In agreement with the earlier Wake et al. (2006) result, the CMASS mass function at the highest masses shows no evolution over $0.45<z<0.7$. The Maraston et al. (2013) analysis is based on 283,819 galaxies spanning $3275 \mathrm{deg}^{2}$.

The question of whether the total mass function evolves has implications for the separate evolution in the numbers of starforming and passive galaxies. At masses below $10^{11} M_{\odot}$, there is broad agreement that the number of "quenched" galaxies increases with time (e.g., Borch et al. 2006; Bundy et al. 2006; Drory et al. 2009; Ilbert et al. 2010; Moustakas et al. 2013), but some controversy remains over whether the star-forming population remains constant (e.g., Ilbert et al. 2010; Moutard et al. 2016b) or declines (e.g., Moustakas et al. 2013), especially at $M_{*}>10^{11}$. A constraint from the total mass function would help distinguish the extent to which star formers shut down and transform into quenched galaxies versus the rate of new arrivals (from lower $M_{*}$ ) that either replenish the star-forming population (e.g., Peng et al. 2010) or add to the increasing number of quiescent galaxies.

The purpose of this work is to study number density evolution at the highest masses using a sample that combines well-understood completeness functions typical of magnitude-limited surveys with large spectroscopic data sets designed to constrain cosmological parameters. In Bundy et al. (2015; hereafter Paper I), we build such a sample by combining SDSS Coadd ugriz photometry in the Stripe 82 region (Annis et al. 2014), reaching $r$-band magnitudes of $\sim 23.5 \mathrm{AB}$, and near-IR photometry in $Y J H K$ bands to 20th magnitude (AB) from UKIDSS-LAS (Lawrence et al. 2007) with 70,000 spec-zs from the SDSS-I/II and BOSS. We refer to the combined data set as the S82-MGC and make it publicly available at http://www.massivegalaxies.com. Paper II in this series, Leauthaud et al. (2016), uses the S82-MGC to investigate the $M_{*}$ completeness limits of the BOSS spec- $z$ samples. The S82-MGC was also used in Saito et al. (2016) to constrain the relationship between high-mass galaxies and their dark matter halos. In this paper, Paper III, we use an $M_{*}$-complete subsample of the S82MGC, comprising $139 \mathrm{deg}^{2}$ and sampling $0.3 \mathrm{Gpc}^{3}$, to measure galaxy mass functions with unprecedented precision at $\log M_{*}$ / $M_{\odot}>11.3$ over $0.3<z<0.65$. Finding no apparent evolution, we place particular emphasis on how scatter in $M_{*}$ measurements, biases resulting from assumptions underlying $M_{*}$ estimates, and other uncertainties limit the interpretation of our results.

A plan of the paper is as follows. We begin in Section 2 by summarizing the key components of the S82-MGC and its construction. Full details can be found in Paper I. The various $M_{*}$ estimates used in this work are described in Section 3. We discuss potential biases in derived mass functions for large samples, including the impact of various forms of measurement scatter, in Section 4. Our results are presented in Section 5, where we study how the adoption of different priors (Section 5.3) and stellar population synthesis models (Section 5.4) affects the degree of evolution we infer. The mass functions of galaxies with different levels of residual SF are presented in Section 5.5 and all results are made available at http://www.massivegalaxies.com. We discuss the significance of our results and their limitations, as well as comparisons to other work, in Section 6. Section 7 provides a summary. Throughout this paper, we use the $\mathrm{AB}$ magnitude system and adopt a standard cosmology with $H_{0}=$ $70 h_{70} \mathrm{~km} \mathrm{~s}^{-1} \mathrm{Mpc}^{-1}, \Omega_{M}=0.3$, and $\Omega_{\Lambda}=0.7$. 

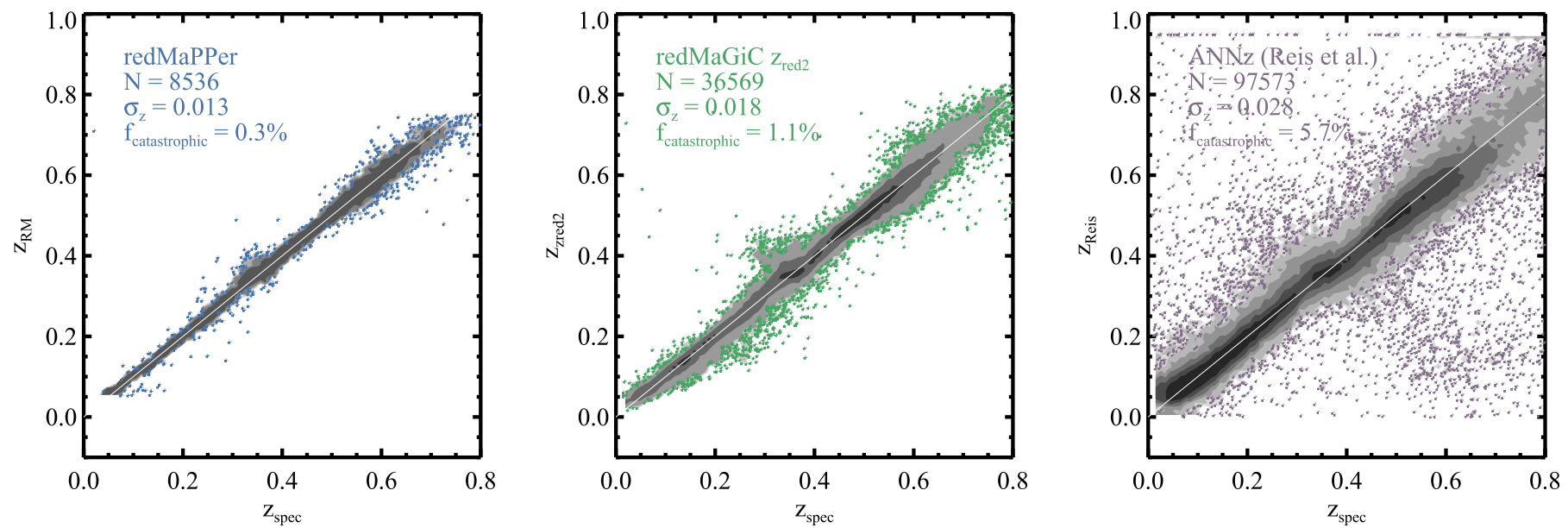

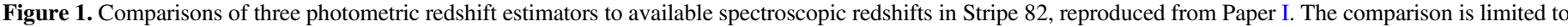

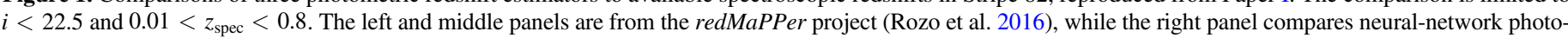

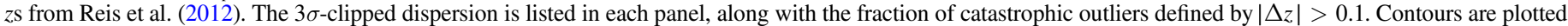

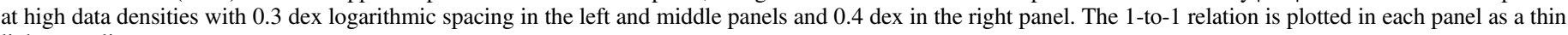
light gray line.

\section{The S82-MGC}

Full details of the S82-MGC catalog construction are presented in Paper I. We summarize key aspects here with a focus on the final UKWIDE sample that we use for our mass function analysis.

\section{1. ugrizYJHK Photometry}

The SDSS Coadd provides the primary source catalog for the s82-MGC. This data set refers to repeated ugriz imaging in Stripe $82\left(-50^{\circ}<\alpha_{\mathrm{J} 2000}<+60^{\circ}\right)$ first presented in Abazajian et al. (2009) and further described in Annis et al. (2014). The point-source 50\% completeness limit for the Coadd is $r \sim 24.4$ (AB). The Coadd photometric catalog is queried as described in Paper I to define a unique sample that is then cross-matched to overlapping near-IR data from the LAS component of UKIDSS (Lawrence et al. 2007) Data Release 8 (DR8). The LAS aims to reach $\mathrm{AB}$ magnitude depths of $Y=20.9, J=20.4, H=20.0$, and $K=20.1$, but we provide field-dependent measures of the achieved depth in the S82-MGC and use these to define an areal footprint that satisfies specific depth requirements in the UKWIDE selection described below.

Point-spread function (PSF)-matched ugrizYJHK photometry in the S82-MGC is obtained with the SYNMAG software (Bundy et al. 2012), which uses SDSS surface brightness profile fits to predict the SDSS $r$-band magnitude that would have been obtained using the same aperture and under the same atmospheric seeing as magnitudes measured in each UKIDSS filter. For total $H$ - and $K$-band magnitudes, which form the basis of our $M_{*}$ estimates, we overcome biases resulting from blended sources in the UKIDSS photometry by building a new flux estimator referenced to the SDSS $z$-band CModelMag magnitude. After correcting for the aperture-matched opticalto-near-IR color (e.g., $(z-K)$ ), we define HallTot magnitudes by adjusting the reported Hall magnitudes to match $\mathrm{CModelMag}_{z}$ on average. For blended sources, which are known to have biased Hall magnitudes, we set the HallTot magnitude to CModelMag and apply the color correction. Further details are given in Paper I.

\subsection{Spectroscopic and Photometric Redshifts}

The SDSS-III program (Eisenstein et al. 2011) BOSS program provides 149,439 spectroscopic redshifts for the S82-MGC. Redshifts from the LOWZ, CMASS, and Legacy samples, as collated in the SDSS-III SpecObj-dr10 catalog, are all included. We combine photometric redshifts from a number of sources to supplement the S82-MGC when spec-zs are not available. For the bright galaxies we study in this work $(i \lesssim 22.5)$, we define $z_{\text {best }}$ to be the spectroscopic measurement, if available. If a photo- $z$ is required, we first check whether the galaxy resides in a cluster with a redshift assigned by the red-sequence Matched-filter Probabilistic Percolation (redMaPPer; Rykoff et al. 2014). Defining $\sigma_{z}$ as the $3 \sigma$-clipped standard deviation of $\Delta z=z_{\text {spec }}-z_{\text {phot }}$ (note that we do not divide by $1+z$ ) and catastrophic outliers as those with $|\Delta z|>0.1$, the redMaPPer photo-zs have $\sigma_{z} \sim 0.02$ and a catastrophic rate of less than $1 \%$. For field galaxies on the red sequence, we adopt estimates from the red-sequence Matched filter Galaxy Catalog (redMaGiC; Rozo et al. 2016). These are only slightly worse in terms of photo- $z$ quality. If neither the redMaPPer nor redMaGiC photo-zs are available, we assign $z_{\text {best }}$ to the neural-network results derived in Reis et al. (2012). The Reis et al. (2012) redshifts have $\sigma_{z} \sim 0.03$ and a 5\% outlier fraction at $z \sim 0.5$. Comparisons of these three photo-z estimators to available spec-zs are presented in Figure 1 and refer the reader to Paper I for further discussion of redshift reliability and completeness.

At $\log M_{*} / M_{\odot}>11.4$ and $z \sim 0.6$, the UKWIDE sample we define below has a spec- $z$ completeness of $80 \%$. Of the remaining galaxies without spec-zs, $\sim 8 \%$ have redMaGiC photo-zs. A roughly equal number have Reis et al. (2012) photo-zs, and a few percent come from redMaPPer. The spec-z completeness improves toward lower redshifts and higher $M_{*}$ (Paper II). We also note that Pforr et al. (2013) found little bias ( $\sim 0.02$ dex) when comparing $M_{*}$ estimates based on photo-zs compared to spec-zs for passive galaxies.

\subsection{The UKWIDE Sample}

The mass functions discussed below are derived using a subset of 517,714 galaxies in the S82-MGC called the UKWIDE sample. The selection criteria are described in detail in Paper I 
Table 1

$M_{*}$ Estimators

\begin{tabular}{|c|c|c|c|c|}
\hline Name & Models & Main Priors & Bursts & $M_{*}$ Scaling \\
\hline$M_{* \mathrm{MGC}}$ & $\mathrm{BC} 03$ & Bundy et al. (2006) & none & reddest band \\
\hline$M_{* \mathrm{MGC}}^{\mathrm{opt}}$ & ВC03 & Bundy et al. (2006) & none & $z$ band \\
\hline$M_{* \mathrm{SED}}$ & FSPS & PRIMUS (Moustakas et al. 2013) & $P_{\text {burst }}=0.2$ & average \\
\hline$M_{* \mathrm{SSED}}^{\mathrm{FSPS}}$ & FSPS & PRIMUS (Moustakas et al. 2013) & none & average \\
\hline$M_{* \mathrm{SSED}}^{\mathrm{BC} 03}$ & $\mathrm{BC} 03$ & PRIMUS (Moustakas et al. 2013) & none & average \\
\hline$M_{* \mathrm{SEDD}}^{\mathrm{Ma} 05}$ & Maraston & PRIMUS (Moustakas et al. 2013) & none & average \\
\hline
\end{tabular}

and include star-galaxy separation, the application of rejection masks in all bands, photometry quality flags, and $5 \sigma \mathrm{YJHK}$ imaging depths of $[20.32,19.99,19.56,19.41]$ in AB magnitudes. The resulting UKWIDE sample spans $139.4 \mathrm{deg}^{2}$ and is complete above $\log M_{*} / M_{\odot} \approx 11.3$ at $z=0.7$.

\section{Stellar Mass Estimates}

As we show in Section 5, systematic uncertainties in $M_{*}$ estimates dominate conclusions about high-mass galaxy growth in the S82-MGC sample. In this section, we present a set of $M_{*}$ estimates based on the same photometric data set and study systematic offsets that arise when different priors, models, and variants of the photometry are used. In Section 5, we will show how $M_{*}$ offsets translate into systematics in the recovered stellar mass function. For comparisons with publicly available BOSS $M_{*}$ estimates, ${ }^{6}$ please see Paper I.

\subsection{The S82-MGC Fiducial $M_{*}$ Estimates}

We recount the description of the S82-MGC $M_{*}$ estimates presented in Paper I. These fiducial $M_{*}$ estimates (we will distinguish them with the label $M_{* \mathrm{MGC}}$ ) are derived using the Bayesian code developed for mass function work in Bundy et al. (2006, 2010). The observed spectral energy distribution (SED) of each galaxy is compared to a grid of 13,440 Bruzual \& Charlot (2003) population synthesis models (BC03), including 16 fixed age values and 35 fixed exponential timescales, $\tau$. Ages are drawn randomly from a uniform distribution between 0 and $10 \mathrm{Gyr}$ and are restricted to less than the cosmic age at each redshift. Values for $\tau$ are also random in the linear range between 0.01 and $10 \mathrm{Gyr}$. No bursts are included, and the dust prescription follows Charlot \& Fall (2000). See Table 1. We assume a Chabrier IMF (Chabrier 2003), $\Omega_{M}=0.3, \Omega_{\Lambda}=0.7$, and a Hubble constant of $70 \mathrm{~km} \mathrm{~s}^{-1} \mathrm{Mpc}^{-1}$.

At each grid point, the reddest band $M_{*} / L_{K}$ ratios (corresponding to the "current" mass in stars and stellar remnants), inferred $M_{*}$, and probability that the model matches the observed SED are stored. This probability is marginalized over the grid, giving an estimate of the stellar mass probability distribution. ${ }^{7}$ We take the median as the final estimate of $M_{*}$. The $68 \%$ width of the distribution provides an uncertainty value that is typically $\sim 0.1 \mathrm{dex}$.

\footnotetext{
6 Tinker et al. (2017) suggested that the "Wisconsin PCA" $M_{*}$ estimates have the smallest measurement uncertainties among available BOSS estimates. While they are compared in Paper I, we do not use them here because they are available only for galaxies with spectroscopic redshifts.

7 Note that we assume the prior grid adequately samples the parameter space of the posterior.
}

\section{2. $M_{*}$ Estimates from iSEDfit}

We also produce $M_{*}$ estimates $\left(M_{* \mathrm{iSED}}\right)$ using the Bayesian iSEDfit package presented in Moustakas et al. (2013). The iSEDfit code has several advantages. In addition to performing a refined grid search of the $M_{*}$ posterior distribution and enabling priors with nonflat probability distributions, iSEDfit can return $M_{*}$ estimates for multiple stellar population synthesis models, including FSPS (Conroy et al. 2009), BC03 (Bruzual \& Charlot 2003), and Maraston (Maraston 2005) models.

The basic set of iSEDfit priors is similar to those used for the $M_{* \mathrm{MGC}}$ estimates and is based on a set (randomly generated for each run of iSEDfit) of 25,000 declining exponential models. The $M_{* \text { iSED }}$ estimates additionally include a prescription for bursts described below. Unlike the $M_{* \mathrm{MGC}}$ models, whose parameters fall on a grid, the parameters for each iSEDfit model vary independently, better sampling the range of each prior. The iSEDfit ages are restricted by the cosmic age at each redshift and drawn linearly from the range $0.1-13 \mathrm{Gyr}$. The exponential $\tau$ prior is drawn from the linear range $0.1-5 \mathrm{Gyr}$. The metallicity and dust assumptions are similar to the $M_{* \mathrm{MGC}}$ estimates. The iSEDfit code is designed to work with flux measurements that we take directly from a conversion of SDSS "Luptitudes" for ugriz and via a transformation to $\mathrm{AB}$ magnitudes for the UKIDSS photometry.

In the case of the $M_{* \mathrm{iSED}}$ fits, stochastic bursts are added randomly to the star formation histories (SFHs). For every $2 \mathrm{Gyr}$ interval over the lifetime of a given model, the cumulative probability that a burst occurs is 0.2 . Each burst's SFH is Gaussian in time, with an amplitude set by $\mathcal{F}_{b}$, the total amount of stellar mass formed in the burst divided by the underlying mass of the smooth SFH at the burst's peak time. The $\mathcal{F}_{b}$ is drawn from the range 0.03-4.0. The allowed burst duration ranges from 0.03 to $0.3 \mathrm{Gyr}$.

Table 1 lists several iSEDfit runs we have performed. The impact of the resulting $M_{*}$ estimates on the derived mass function is discussed in Sections 5.3 and 5.4.

\subsection{Optical versus Near-IR Photometry}

Providing photometric coverage in the near-IR, which is more sensitive to older stellar populations that typically dominate $M_{*}$, was one of the motivations for assembling the S82-MGC (Bundy et al. 2015). We can test the impact of nearIR photometry by comparing the standard $M_{* \mathrm{MGC}}$ estimates, which are based on ugrizYJHK, to those using solely the ugriz bands (we label these $M_{* \text { MGC }}^{\text {opt }}$ ). We use the $M_{* \text { MGC }}$ mass estimator in both cases. Figure 2 tracks the mass difference, $M_{* \mathrm{MGC}}-M_{* \mathrm{MGC}}^{\mathrm{opt}}$, as a function of several parameters. For masses above $M_{* \mathrm{MGC}}>10^{9} M_{\odot}$, the top left panel reveals a small offset of -0.07 dex with a scatter of 0.06 dex but no strong dependencies on $M_{* \mathrm{MGC}}$. The difference in mass estimates systematically changes for lower-redshift galaxies 

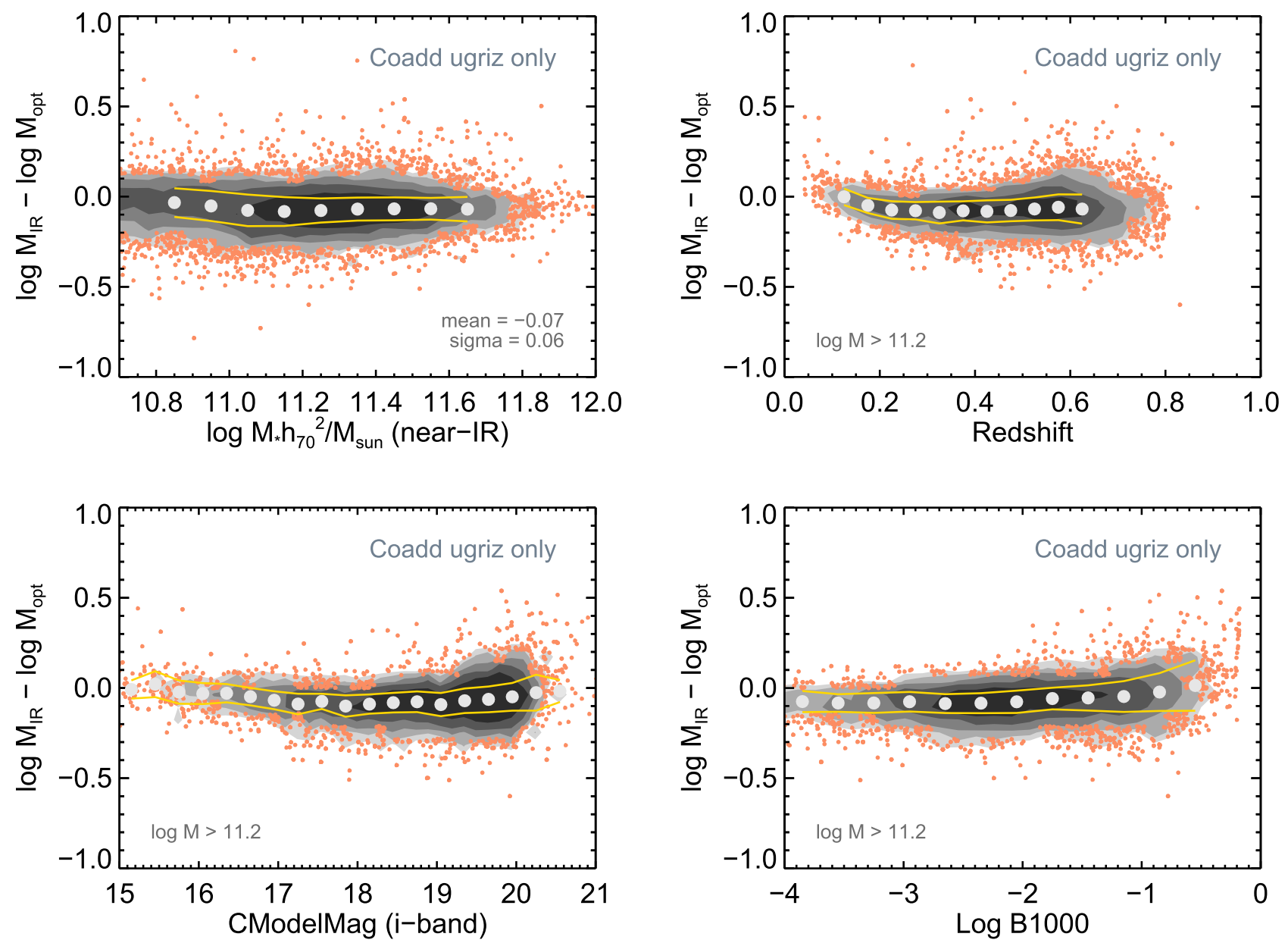

Figure 2. Comparison of $M_{* \mathrm{MGC}}$ estimates from SED fitting applied to optical photometry only (ugriz) with those from optical+near-IR photometry (ugrizYJHK). For near-IR masses, $M_{*}$ is estimated by scaling the determined $M / L$ in the observed reddest band by the observed luminosity in that band as measured using the KHallTot magnitude. For optical masses, the observed-frame $z$-band $M / L$ is scaled by SDSS Coadd $z$-band CModelMag. The optical-near-IR $M_{*}$ difference is plotted as a function of near-IR $M_{*}$ (top left), redshift (top right), $i$-band CModelMag (bottom left), and the birth parameter, $b_{1000}$, a measure of the inferred SFR averaged over the last $1000 \mathrm{Myr}$ compared to the SFR averaged over the galaxy's lifetime.

with apparent magnitudes brighter than $\sim 17 \mathrm{AB}$ (top right and bottom left panels). The final panel in Figure 2 investigates the dependence on $b_{1000}$, a measure of recent SF composed of the ratio of the star formation rate (SFR) averaged over the last 1000 Myr to the average SFR over the galaxy's lifetime. This panel shows that across mass and redshift, galaxies in the S82MGC with higher $b_{1000}$ values, implying more recent SF, deviate from the -0.07 dex offset that defines $M_{* \mathrm{MGC}}-M_{* \mathrm{MGC}}^{\mathrm{opt}}$ for most of the sample. These galaxies show offsets that are $\sim 0.1$ dex larger and have $\log M_{*} / M_{\odot} \lesssim 11.5$.

The systematic differences in $M_{*}$ that are evident in Figure 2 arise from two sources. First, near-IR photometry provides additional constraints on galaxy SEDs that should yield better estimates of mass-to-light $(M / L)$ ratios. Errors from photometric matching across many bands could also degrade the SED fit quality, however. Figure 2 shows that for the redshifts relevant to this work $(z>0.2)$, including near-IR constraints has little or no effect on $M_{*}$ estimates, suggesting that ugriz photometry alone provides estimates for massive galaxies at $z<0.8$ similar to those of optical+near-IR photometry. Perhaps not surprisingly, however, the role of near-IR data becomes important for the modest number of massive galaxies with recent SF (bottom right panel). Here, assuming that the near-IR masses are more accurate, the optical-only estimates may be biased low by -0.1 dex, with deviations as high as -0.5 dex in individual cases.

The second factor behind the systematic differences in Figure 2 is the use of different total flux estimators. The $M_{* \text { MGC }}$ estimates are the result of multiplying the $M / L$ derived for the observed-frame $K$ band $^{8}$ by KHallTot, a nonparametric total magnitude estimate. As discussed in Paper I, the KHallTot measurements are less biased by blended sources compared to other flux estimators in the UKIDSS photometry. However, KHallTot must be adjusted globally to match the $z$-band CModelMag estimates. The $M_{* M G C}^{\text {opt }}$ estimate, on the other hand, is the direct product of the observed-frame $z$-band $M / L$ and the z-band CModelMag. The CModelMag estimator combines total flux measures from SDSS-derived 2D fits of an exponential and a de Vaucouleurs surface brightness profile. Differences in the way CModelMag and HallTot account for the "total light" in a surface brightness profile can therefore impact the $M_{*}$ measurements.

Figure 3 explores this by comparing the flux corresponding to KHallTot to that from the $z$-band CModelMag $z$ as a function of CModelMag (left panel). The effect of $(z-K)$ color (derived from PSF-matched photometry) has been

In rare cases, the $H$ band is used when the $K$ band is not available. 

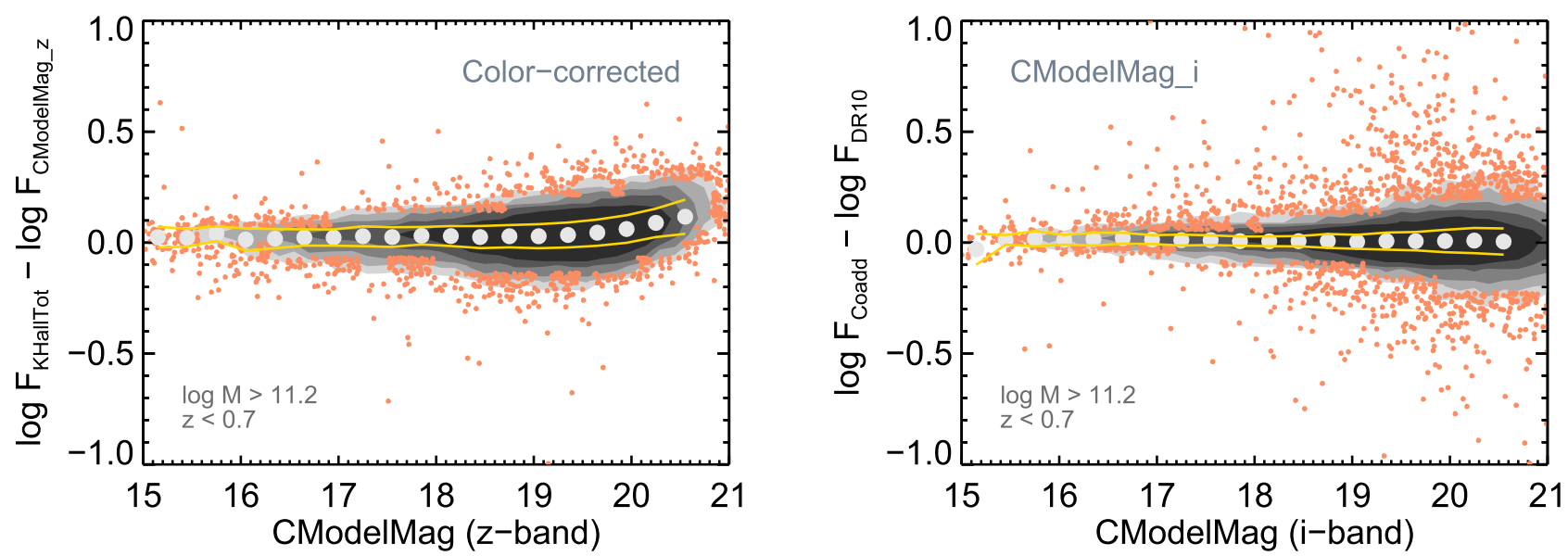

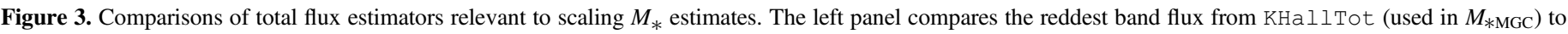

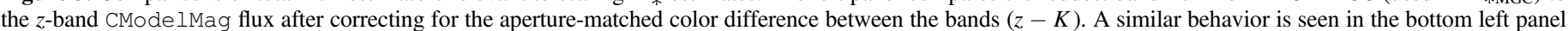

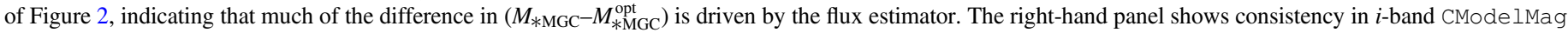
measurements between the Coadd photometry and single-epoch DR10 (the $z$ band shows similar behavior).

removed. The flux difference remains flat until CModelMag $z$ $\sim 19 \mathrm{AB}$, at which point the near-IR flux estimator grows slightly in comparison to CModelMag $z$ KHall Tot is $0.1-0.2$ dex brighter at the faintest magnitudes in the sample. This trend seems to be expressed in the direct $M_{*}$ comparison against CModelMagi $_{i}$ (Figure 2, bottom left panel). But systematic differences in total flux cannot explain the increasing $M_{*}$ discrepancy at CModelMag $i<17 \mathrm{AB}$ and, correspondingly, $z<0.2$. In this very bright regime, changes in the $M / L$ inferred from SED fits to the multiband photometry must be responsible. We do not pursue these $M_{*}$ offsets further because, for the mass function analysis that follows, we restrict ourselves to higher redshifts.

A final test is provided in the right-hand panel of Figure 3, which compares CModelMag estimates from the SDSS Coadd (used in the S82-MGC) to those from the single-epoch SDSS photometry. There is an expected increase in scatter at fainter magnitudes (because the Coadd is much deeper), but no evidence for systematic trends. Given that CModelMag $i$ is the dominant, and often sole, total magnitude used to normalize other $M_{*}$ estimates provided by the BOSS team, the good agreement shown here makes the Coadd-based S82-MGC a valuable anchor for understanding $M_{*}$ systematics in studies exploiting the full BOSS data set (e.g., Maraston et al. 2013).

\section{Methods: Number Density Distributions in Large-volume Surveys}

One of the goals of this paper is to use the S82-MGC to explore the new "large-volume" regime for complete studies of galaxy number density distributions, such as the mass and luminosity functions. For samples spanning more than $\sim 100 \mathrm{deg}^{2}$ and a significant redshift baseline, several considerations arise. An obvious point is that making use of the statistical precision afforded by large volumes requires careful control of the error budget. Ideally, we would restrict ourselves to using only spectroscopic redshifts for this reason, but in the near term, obtaining spec-zs for the tens of millions of sources that current imaging surveys now detect (e.g., to $i<23 \mathrm{AB}$ ) is infeasible.

Even if we limit ourselves (as we do in the next section) to brighter subsamples where spectroscopic follow-up is possible, we are left with the challenge of determining the completeness of the sample at a level of precision on par with that of the number density measurements themselves. One must either additionally commit significant spectroscopic resources to defining the completeness limit (i.e., "throwing away" a large number of hardearned spec-zs), estimate the completeness by applying the selection criteria to simulated samples (see Maraston et al. 2013), or turn to photometric redshifts, as we do in this work to supplement redshift information where the spec-zs are incomplete. In the sample we use below, the fraction of galaxies with $\log M_{*} / M_{\odot}>11.4$ (Chabrier IMF) and $z<0.6$ that require photo-zs is roughly $20 \%$ (Leauthaud et al. 2016).

The introduction of photo-zs adds sources of both random and systematic error that must be accounted for (e.g., Etherington et al. 2017). At the same time, a new tool for diagnosing such errors becomes available when the expected, random statistical fluctuations (including sample variance) are negligible. That tool is essentially a prior that dictates that the shape and normalization of actual number density distributions should evolve smoothly with redshift. A stronger version would assume that evolution in the average properties of the galaxy distribution is both smooth and monotonic. A particular redshift bin, for example, with a clear excess number density can be a signpost of systematic errors that preferentially affect those redshifts.

\subsection{Biases from Photometric Redshifts}

While the mass functions derived here rely on a sample with $\gtrsim 80 \%$ spec- $z$ completeness, the use of photo- $z$ s can introduce errors in a number of ways. These include biases in the binned redshift distribution itself, scatter in the luminosity distance used to normalize $M_{*}$, and errors on the recovered rest-frame SED.

To first order, photo-zs introduce a Gaussian redshift uncertainty, blurring out structure in the true redshift distribution and creating contamination between adjacent redshift bins. The effect of contamination is reduced as the bin size increases above the $1 \sigma$ photo- $z$ uncertainties. If the photo- $z$ uncertainty depends on redshift, the bin-to-bin contamination will vary with $z$ as well. Defined $z$ bins at the limits of the full range accessible will also have true redshift distributions that are asymmetric. These effects are typically small, because photo- $z$ uncertainties of $\sigma_{z} \sim$ 0.03-0.07 can often be achieved and are usually smaller than the redshift baselines probed $(\Delta z>0.3)$. These uncertainties also depend weakly on $z$ across most samples. Finally, biases in the mean photo- $z$ are often much smaller than $\sigma_{z}$. 
The larger impact of roughly Gaussian photo- $z$ uncertainties is the contribution of an additional random error on the $M_{*}$ (or $L$ ) estimates as a result of their dependence on the now-uncertain luminosity distance. The resulting photo-z-induced scatter in $\log M_{*}$, which we refer to as $\sigma_{M_{*}, z}$, as a result of the photo- $z$ uncertainty, $\sigma_{z}$, can be estimated as $\sigma_{M_{*}, z} \approx \sigma_{z} / z$. Among the worst photo-zs $\left(\sigma_{z}=0.04\right)$ in the S82-MGC at $z \approx 0.6$, for example, the photo- $z$ uncertainty adds 0.07 dex in quadrature to the $M_{*}$ errors, which exhibit $\sigma_{M_{*}}=0.1-0.2$ dex when $z$ is perfectly known. We will discuss how random errors in $M_{*}$ can be addressed in the next section.

In addition to making luminosity distances more uncertain, the photo- $z$ error shifts the inferred rest-frame wavelength of the SED, thereby degrading the quality of the fit and derived $M / L$ ratio. This effect is small. Tests applied to the S82-MGC neuralnetwork photo-zs, which have $\sigma_{z} \approx 0.02-0.03$, indicate that this rest-frame color uncertainty adds 0.02 dex in quadrature to $\sigma_{M_{*}}$.

A more subtle but extremely important problem occurs when the photo- $z$ scatter increases over a specific range in redshift. A look at the photo- $z-$ spec- $z$ comparison in Figure 1 shows an often-seen degradation in photo- $z$ quality at $z \approx 0.35$ that corresponds to the $4000 \AA$ break falling between the $g$ and $r$ bands. With only a few thousand spec-zs to compare against, this feature is hardly noticeable. Furthermore, because the additional scatter appears roughly symmetric, it is tempting to believe that any effect on the mass or luminosity function would cancel out.

When tens of thousands of spec-zs are available, as in the $\mathrm{S} 82-\mathrm{MGC}$, this photo- $z$ feature reveals itself to be much more prominent, with a noticeable tail. The key point is that the direction of photo- $z$ scatter can have a profound impact on derived number density functions. Upscattering yields a greater distance for a galaxy, shifting it into a higher photo- $z$ bin and assigning a higher $M_{*}$ or $L$ than it deserves. Because more massive and intrinsically luminous galaxies are significantly rarer than their low-mass counterparts, upscattering can create a significant bias in the reported number density evolution. Even when photo- $z$ downscattering is symmetric, it has a less significant impact, because the true number of lower-mass galaxies significantly outweighs the number of contaminants.

Similar arguments apply to the location of catastrophic photo- $z$ outliers. For these and other kinds of photo- $z$ behavior, it may be possible to influence photo- $z$ codes so that they fail in preferred ways. In others, the choice of redshift bins can be designed to avoid regions of worrisome contamination. It may also be possible to model photo- $z$ effects and account for them, although this is beyond the scope of the current paper.

\subsection{Accounting for Scatter in $M_{*}$ or $L$}

Even with spec- $z$-only samples, random errors in the $M_{*}$ (or $L$ ) estimates introduce Eddington bias in the derived galaxy mass functions as a result of the steep decline in the number of galaxies at the bright end. The contamination from intrinsically lower-mass galaxies scattering upward outweighs the downscattering of higher-mass galaxies because there are many more lower-mass galaxies subject to random $M_{*}$ errors. The result is that scatter in $M_{*}$ "inflates" the observed mass function at the high-mass end, a bias that becomes worse as the scatter increases (e.g., from additional photo-z-related error terms). The goal in this work is to study evolution in the number density distribution. If the scatter term evolves with redshift, as would be expected because the $\mathrm{S} / \mathrm{N}$ of observations degrades with redshift, then the observed evolution may be biased by the changing importance of Eddington bias.

If the various $M_{*}$ error terms can be estimated, one solution is to perturb the final $M_{*}$ values until the scatter is uniform across the sample. For the S82-MGC, we estimated the $M_{*}$ error for each galaxy resulting from the uncertainty in the total magnitude estimate (which normalizes $M_{*}$ ). If no spec- $z$ was available, we added in quadrature to this value the expected $M_{*}$ error resulting from the assigned photo- $z$ (according to the redshift-dependent performance of the associated photo- $z$ estimator as compared to spec- $z \mathrm{~s})$. Based on the maximum errors obtained for galaxies in our sample, we set a target for the final uncertainty of all galaxies at $\sigma_{M_{*}}=0.115$ dex. We used a Gaussian kernel with a width equal to the difference in quadrature between this target error and the estimated error for each galaxy to describe the degree of perturbation required to make the final scatter uniform for each $M_{*}$ estimate. In other words, random draws from these kernels were added to each $M_{*}$ estimate to obtain a set of perturbed $M_{*}$ estimates. The scatter resulting from magnitude and redshift errors is uniform for these perturbed values. We did not account for the additional error term that arises from model-fitting uncertainties in $M_{*}$ because these indicate no redshift dependence and are, themselves, uncertain. The resulting mass functions derived with the perturbed sample of $M_{*}$ values is presented in Section 5.2.

A second solution to accounting for a varying Eddington bias is to assume an intrinsic shape for the $M_{*}$ or $L$ function and forward-model the data while accounting for the estimated uncertainties (e.g., Moutard et al. 2016a). As described in Leauthaud et al. (2016), we consider the same sources of error on a per-galaxy basis as described above. We assume a double Schechter function (Baldry et al. 2008) of the form

$$
\begin{aligned}
& \phi\left(M_{*}\right)=(\ln 10) \exp \left[-\frac{M_{*}}{M_{0}}\right] \\
& \times\left\{\phi_{1} 10^{\left(\alpha_{1}+1\right)\left(\log M_{*}-\log M_{0}\right)}+\phi_{2} 10^{\left(\alpha_{2}+1\right)\left(\log M_{*}-\log M_{0}\right)}\right\},
\end{aligned}
$$

where $\alpha_{2}>\alpha_{1}$ and the second term dominates at the low-mass end. We generate Monte Carlo realizations of this function that sample various parameter ranges as described below. A mock sample is drawn from each realization, and the individual scatter terms are added to $M_{*}$. The mock samples are binned identically to the data and compared to the observed number density distributions in an iterative approach that allows the input parameters to be constrained.

\subsection{Sample Variance}

Large-volume surveys significantly mitigate the impact of sample variance (often called "cosmic variance") that arises from large-scale fluctuations in the spatial distribution of galaxies in the universe (see Moster et al. 2011). Stringer et al. (2009) showed, for example, that galaxy surveys spanning more than $\sim 100 \mathrm{deg}^{2}$ are needed to overcome sample variance on measurements of evolution in the mass function at $z<1$.

An estimate of the sample variance in the S82-MGC can be made using an abundance-matched mock catalog (see Leauthaud et al. 2016). The volume of the mock, $1 \mathrm{Gpc}^{3} h^{-3}$, can be divided into multiple subvolumes corresponding to 0.1-width redshift slices of the $139.4 \mathrm{deg}^{2}$ S82-MGC. In each redshift bin, we study the mass function distribution contributed from four to five mock subvolumes with a similar volume to Stripe 82. Additional 
observational errors, as well as redshift evolution, are ignored. In the $0.3<z<0.4$ bin $\left(0.02 \mathrm{Gpc}^{3} h^{-3}\right)$, this experiment yields a $1 \sigma$ error of 0.014 dex at $\log M_{*} / M_{\odot} \sim 11.0$, rising to 0.02 dex at $\log M_{*} / M_{\odot} \sim 11.6$. For $0.3<z<0.4\left(0.04 \mathrm{Gpc}^{3} h^{-3}\right)$, the value is 0.008 dex at $\log M_{*} / M_{\odot} \sim 11.0$ but remains at 0.02 dex for $\log M_{*} / M_{\odot} \sim 11.6$. The errors rise further toward $0.1 \mathrm{dex}$ at $\log M_{*} / M_{\odot} \sim 12.0$, where Poisson errors from the limited number of massive mock halos also contribute.

Our adopted sample variance and Poisson error estimates come from bootstrap resampling the derived number densities. We divide the S82-MGC into 214 roughly equal area regions and recompute number density functions after resampling with replacement. This technique yields results consistent with those of the mock catalog analysis with the benefit of allowing us to map covariance matrices (see the Appendix) that facilitate comparisons to theoretical predictions (see Benson 2014). Given the correlations in the large-scale clustering of dark matter halos across halo mass, one expects strong covariance across $M_{*}$ and $L$ in galaxy number densities as inferred from this analysis.

\section{Results}

\subsection{Assumption-averaged Estimate of the Stellar Mass Function}

We begin with estimates for the evolving galaxy $M_{*}$ functions derived from the S82-MGC data set after averaging a set of four $M_{*}$ estimates made using different sets of priors and stellar population models. In the sections that follow, we examine how these functions change under different assumptions. Following Bundy et al. (2015), we use the most accurate redshift available for each galaxy, $z_{\text {best }}$, which is dominated by spec-zs for the majority of the sample. Given subtle differences among $M_{*}$ estimates, which we investigate below, we define the "assumption-averaged" mass function from the average ${ }^{9}$ of results from four different sets of nine-band $M_{*}$ estimates: $M_{* \mathrm{MGC}}$ (original S82-MGC estimates), $M_{* \mathrm{iSED}}$ (FSPS with bursts), $M_{* \mathrm{iSED}}^{\mathrm{BC03}}$ (BC03 models, no bursts), and $M_{* \mathrm{iSED}}^{\mathrm{Ma} 5}$ (Maraston models, no bursts). These four estimates encompass the range of $M_{*}$ values obtained by adopting currently uncertain priors. Without more information about how to set accurate priors or which models to favor, the assumption-averaged result represents a compromise among differing approaches.

Figure 4 plots the "as observed" results with shaded regions corresponding to bootstrap errors (i.e., both Poisson and sample variance errors are included). No $M_{*}$ scatter normalization has been applied. The redshift bins are defined as $z=$ $[0.3,0.4],[0.4,0.5],[0.5,0.6],[0.6,0.65]$, and we indicate the $M_{*}$ completeness limit of $\log M_{*} / M_{\odot}=11.3$ derived in Bundy et al. (2015) with the vertical dotted line.

We have also forward-modeled the observed number densities to account for Poisson errors and scatter in $M_{*}$ uncertainties arising from SED fitting (fixed at $0.07 \mathrm{dex}$ ), photo- $z$ uncertainty for galaxies without spec-zs, and total flux errors, all of which are assumed to be Gaussian and are added in quadrature on a per-galaxy basis. A set of intrinsic fitted models are indicated as dotted lines with the same $z$-dependent colors. Because the modeling involves random draws from estimated error distributions, the intrinsic models can vary from run to run with a scatter consistent with the error bars indicated on the raw mass functions in Figure 4.

\footnotetext{
9 In practice, the average number densities are computed by binning a concatenated array of four different sets of $M_{*}$ estimates and dividing by four times the corresponding volume of each redshift slice.
}

The modeling assumes the double Schechter form described in Section 4.2 and allows $\phi_{1}, \phi_{2}$ and $M_{0}$ to vary while fixing $\alpha_{1}=-0.1$ and $\alpha_{2}=-1.0$. The choice of faint-end slopes and derived model parameters is degenerate and is not meant to convey physical insight. We have selected this model form because it accurately describes the data under our forwardmodeling analysis. The results are given in Table 2. Tabulated data points are available from http://www.massivegalaxies.com.

As in Paper II, we can extend our characterization of galaxy stellar mass function to lower $M_{*}$ by including data from other surveys. For $M_{*}>10^{10.4} M_{\odot}$ but below the completeness limit of the S82-MGC, our forward-model fits include results from the PRIMUS mass functions (Moustakas et al. 2013) observed at similar redshifts. While the PRIMUS data do not impact the derived mass functions at $M_{*}>10^{11.3} M_{\odot}$, their inclusion makes the intrinsic mass functions in Table 2 broadly representative of the galaxy population with $M_{*}>10^{10.4} M_{\odot}$ and $z<0.6$.

Within the statistically tight error bars from the S82-MGC sample, we detect no redshift evolution over most of the mass range probed. At the lowest masses, there is a hint of positive growth (either in $M_{*}$ at fixed number density or in number at fixed $M_{*}$ ), although this likely reflects incompleteness at the faint end, which would produce a similar trend. We will discuss the appropriate confidence level of our no-evolution result in Section 6.1.

The gray data points in Figure 4 represent the $z \approx 0$ mass function from SDSS as derived by Li \& White (2009). With smaller redshift surveys, comparisons to SDSS have been subject to systematic offsets in the assumptions between $M_{*}$ estimates (e.g., Moustakas et al. 2013). In the S82-MGC, however, there are sufficient numbers of galaxies that overlap with the Li \& White (2009) sample that we can characterize systematic offsets in $M_{*}$ and statistically remove them. The Li \& White (2009) $M_{*}$ estimates are taken from the Petrosian Kcorrect quantities, which use $\mathrm{BC} 03$ models and are provided in the New York University Value Added Catalog (NYUVAGC; Blanton \& Roweis 2007). After adjusting the Hubble parameter to $h=72$, we compare these $M_{* \text { VAGC }}$ values to $M_{* \mathrm{MGC}}$ for 3515 galaxies with $11.0<\log M_{*} / M_{\odot}<11.8$ and $0<z<0.2$. We fit a line to the mass difference $\left(\Delta \log M_{*}=\right.$ $\left.M_{* \mathrm{VAGC}}-M_{* \mathrm{MGC}}\right)$ as a function of $M_{* \mathrm{VAGC}}$, referenced to $\log M_{* \mathrm{VAGC}} / M_{\odot}=11.3$, and adjust the Li \& White (2009) mass functions to account for the difference. The fit's zeropoint offset is 0.1 dex with a slope of -0.08 .

Finally, we convolve the SDSS Li \& White (2009) mass function with additional scatter in $M_{*}$ to approximate the Eddington bias in the S82-MGC that results from larger photometric errors in both the total magnitudes and colors of the higher- $z$ sample. The convolution follows the approximation given in Behroozi et al. (2010). With typical total $K$-band uncertainties of $0.05 \mathrm{mag}$, a reasonable estimate for the additional $M_{*}$ scatter in the S82-MGC is $\sigma=0.12$ dex. Applying $\sigma=0.12$ dex to the SDSS mass function results in the solid gray data points plotted in Figure 4. The mass-adjusted Li \& White (2009) mass function with this additional scatter falls almost directly on the S82-MGC results, with a hint of lying on the more massive side of the S82-MGC mass functions.

However, our uncertainty in the correct amount of additional scatter to apply limits a precise comparison between the S82-MGC and SDSS $z \approx 0$ mass functions. If we slightly reduce the applied scatter to $\sigma=0.09$, still a reasonable approximation to the true value, the resulting SDSS mass function falls significantly $(0.1-0.2$ dex $)$ below the S82-MGC results. We conclude that the 


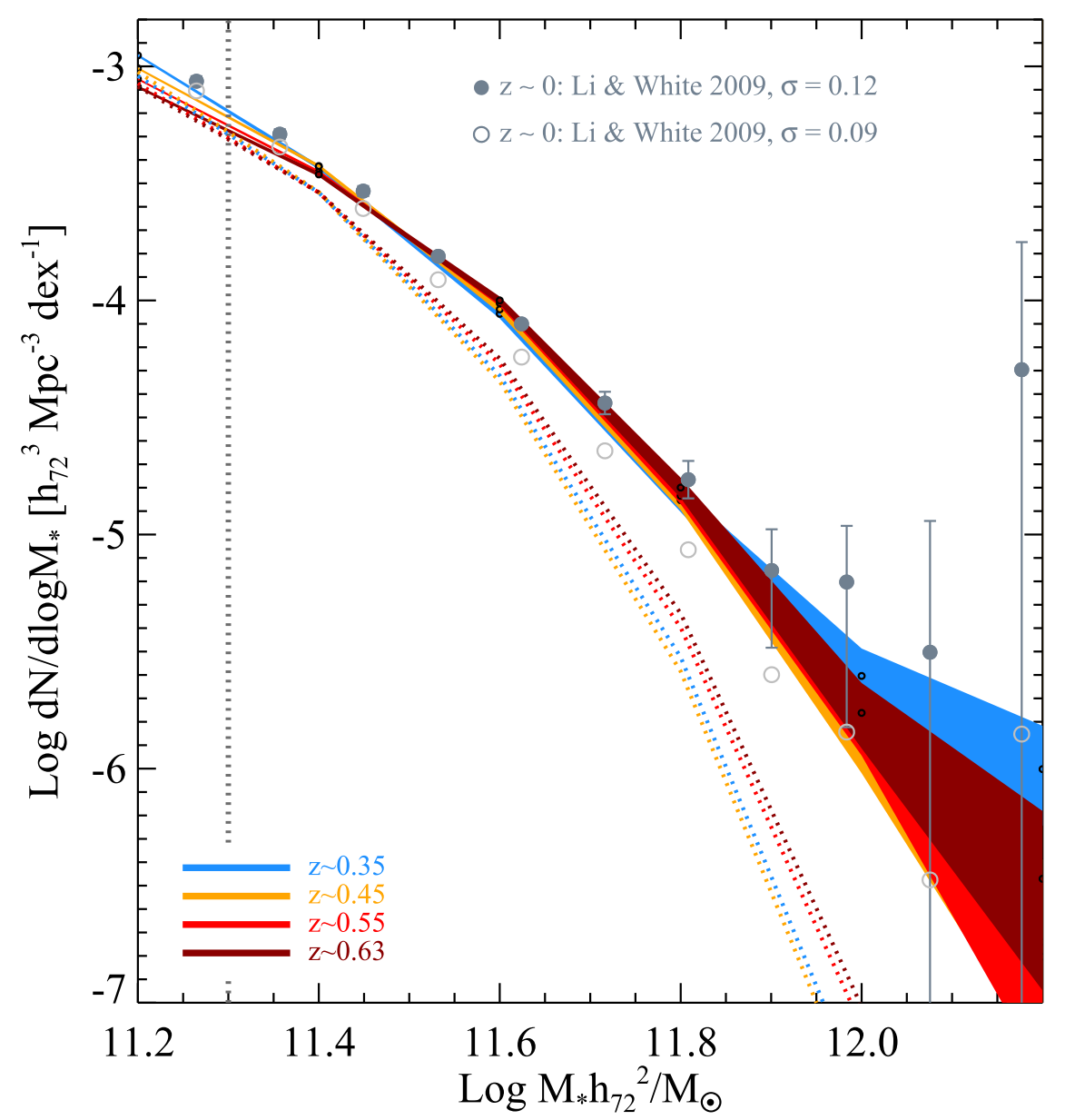

Figure 4. Assumption-averaged estimated $M_{*}$ function made by combining four separate $M_{*}$ estimators using different models and prior assumptions. Shaded regions indicate Poisson errors only. The estimated $M_{*}$ completeness is indicated by a vertical dotted line at $\log M_{*} / M_{\odot}=11.3$. Gray data points show the $z \approx 0$ SDSS MF and associated errors from Li \& White (2009) after scaling their $M_{*}$ estimates to the $M_{* \mathrm{MGC}}$ (for galaxies in common) and convolving with two levels of scatter, as indicated. Forward-modeling results, which aim to account for (and thereby remove) biases caused by measurement scatter, are shown with dotted lines. These fits are subject to additional uncertainties in the assumed functional form and the modeling of various sources of scatter.

S82-MGC and SDSS $z \approx 0$ mass functions are in agreement, with no detected differences at the 0.1 dex level. This comparison includes a careful attempt to normalize the $M_{*}$ estimates, a process that should also remove biases from different estimators of total luminosity (e.g., Bernardi et al. 2013). However, a more precise treatment of $M_{*}$ scatter, let alone further assessments of systematic biases in $M_{*}$ estimates (see below), is needed before these data sets can be used to measure growth in $M_{*}$ with the needed sub-10\% level precision.

\subsection{Scatter-normalized Mass Functions}

The assumption-averaged S82-MGC mass functions, both in raw form and from forward-model fitting, show no evidence of redshift evolution. While the forward model should account for the effect of scatter, we provide a second test here using perturbed $M_{*}$ estimates. Following the methodology in Section 4.2 , we perturb the $M_{*}$ values in order to normalize the scatter from photo-zs and luminosity errors, aiming for a uniform $\sigma_{M_{*}}$ uncertainty resulting from these two terms of 0.115 dex. The mass functions using these perturbed $M_{*}$ values are shown in Figure 5. As expected, the number densities are inflated with respect to Figure 4, but in a way that impacts all redshift bins equally. The fraction of photo-zs is relatively small in the S82-MGC and increases somewhat toward lower redshifts. The combination of photo- $z$ and luminosity error in the $M_{*}$ uncertainties is thus roughly balanced as a function of redshift in the raw mass functions presented in Figure 4.

Confirming the results from the previous section, no redshift evolution is apparent using the scatter-normalized $M_{*}$ values from the combined set of mass estimates.

\subsection{Dependence on Priors}

The mass function results from the previous sections average estimates from four different sets of $M_{*}$ measurements that include different SFH priors and stellar synthesis models. These assumption-averaged mass functions show no evidence for redshift evolution, but redshift differences do appear when specific sets of $M_{*}$ estimates are used, underlining the importance of systematic errors in $M_{*}$ values when measuring precise growth rates at these masses.

We find that different priors in the SFH lead to the largest discrepancies both in terms of absolute $M_{*}$ differences and, more importantly, in terms of the implied redshift evolution. Figure 6 shows raw mass functions based on three sets of $M_{*}$ estimates: $M_{* \mathrm{MGC}}, M_{* \mathrm{iSED}}$, and $M_{* \mathrm{iSED}}^{\mathrm{FSPS}}$. The $M_{* \mathrm{MGC}}$ mass functions (left panel) exhibit an apparent decrease of $0.1 \mathrm{dex}$ in the $M_{*}$ values of massive galaxies over the sampled redshift range. Results with bursty SFHs $\left(M_{* \text { iSED }}\right.$; middle panel) show a mild reversal of this trend, while the burst-free $M_{* \text { iSED }}^{\text {FSPS }}$ estimates (right panel) imply little to no evolution. We show in the next section that the 
Table 2

Intrinsic Mass Function Shape Parameters from Forward Modeling

\begin{tabular}{|c|c|c|c|c|c|}
\hline Redshift & $\log _{10}\left(\phi_{1} / \mathrm{Mpc}^{-3} \mathrm{dex}^{-1}\right)$ & $\log _{10}\left(\phi_{2} / \mathrm{Mpc}^{-3} \mathrm{dex}^{-1}\right)$ & $\log _{10}\left(M_{0} / M_{\odot}\right)$ & $\alpha_{1}$ & $\alpha_{2}$ \\
\hline$[0.30,0.40]$ & $-5.92 \pm 0.03$ & $-2.50 \pm 0.02$ & $10.88 \pm 0.01$ & -0.10 & -1.00 \\
\hline$[0.40,0.50]$ & $-6.00 \pm 0.03$ & $-2.46 \pm 0.01$ & $10.87 \pm 0.01$ & -0.10 & -1.00 \\
\hline$[0.50,0.60]$ & $-5.63 \pm 0.01$ & $-2.60 \pm 0.01$ & $10.91 \pm 0.01$ & -0.10 & -1.00 \\
\hline$[0.60,0.65]$ & $-5.90 \pm 0.02$ & $-2.64 \pm 0.01$ & $10.93 \pm 0.01$ & -0.10 & -1.00 \\
\hline
\end{tabular}

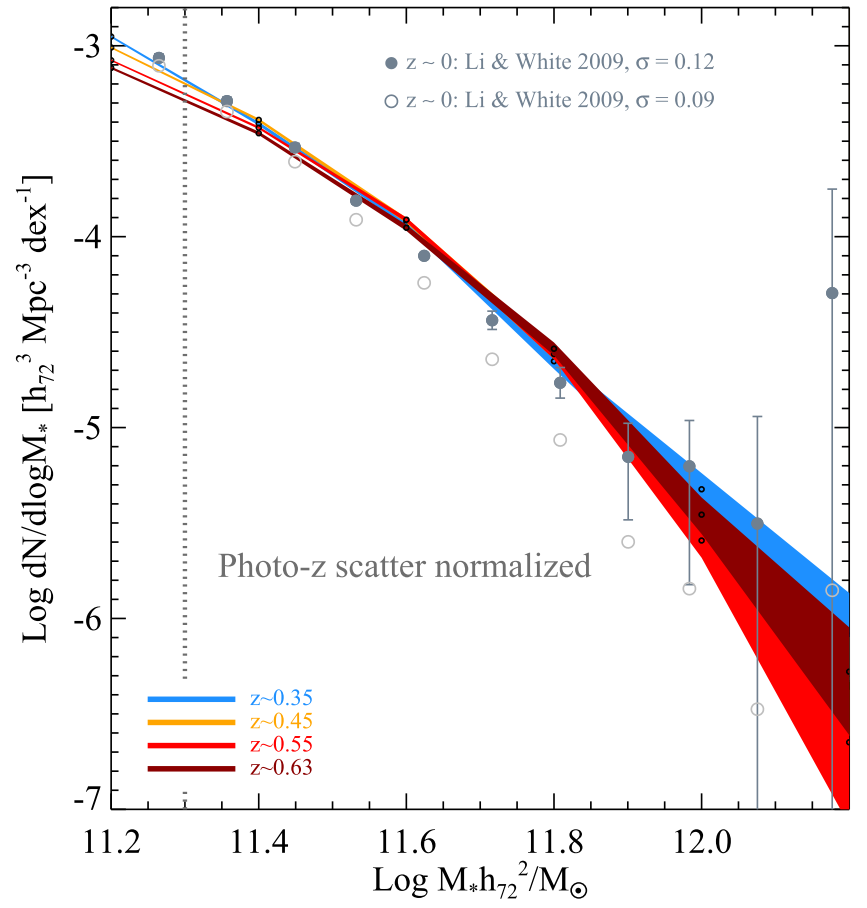

Figure 5. Mass functions as in Figure 4 but using $M_{*}$ estimates that have been perturbed to exhibit uniform photo- $z$-induced scatter across the redshift range probed. The additional scatter causes an Eddington bias that inflates the derived number densities compared to Figure 4, but this bias affects all redshift bins equally. The scatter-normalized mass functions thus remain consistent with no evolution, confirming the results of the forward-model fits in Figure 4.

impact on evolutionary signals of different stellar synthesis models is modest, so while $M_{* \mathrm{MGC}}$ estimates are based on $\mathrm{BC} 03$ and the other estimates in Figure 6 on FSPS, we ascribe most of the differences observed to SFH priors.

The bursty $M_{* \text { iSED }}$ mass functions (middle panel) not only suggest mild growth in $M_{*}$ with time-the opposite conclusion of the $M_{* \mathrm{MGC}}$ results in the left-hand panel—but feature a more significantly elevated result at high masses in the $z \approx 0.35$ bin compared to the $M_{* \text { iSED }}^{\text {FSPS }}$ number densities (right panel). The $M_{* \text { iSED }}^{\text {FSPS }}$ results are consistent with no evolution over the majority of the mass range probed. The difference at higher masses likely reflects the impact of priors that control the burst histories.

While we leave a detailed investigation of the role of specific SFH priors and their optimization for this sample to future work, we conclude from Figure 6 that the resulting uncertainties introduce a systematic error of 0.03 dex in the $M_{*}$ growth histories that we can determine from our combined assumptionaveraged mass function (absolute $M_{*}$ differences can be somewhat larger). This level of systematic uncertainty resulting from $M_{*}$ modeling is similar to that cited by Moustakas et al. (2013).

\subsection{Impact of Stellar Synthesis Models}

Figure 7 allows us to evaluate how three choices for the stellar population models underlying the iSEDfit $M_{*}$ estimates impact constraints on stellar mass growth. In all cases, models without bursts are compared. The FSPS $M_{* \text { iSED }}^{\text {FSPS }}$ mass functions are repeated from Figure 6 in the left-hand panel. Mass functions based on BC03 masses, $M_{* \mathrm{iSED}}^{\mathrm{BCO}}$, are shown in the middle panel, while the right-hand panel uses $M_{* \mathrm{iSED}}^{\mathrm{Ma} 05}$, based on models from Maraston (2005).

From one panel to the next, absolute differences in the mass estimates manifest in changes to the derived set of mass functions. But the implied differential redshift evolution within each panel is nearly identical and again consistent with no detectable growth with redshift. At least among the set of stellar population synthesis models used here, model differences are less important than SFH priors in affecting conclusions about the average growth rates in massive galaxy populations.

\subsection{Dependence on Star Formation History}

In this section, we partition the high-mass S82-MGC galaxy population into different subsamples based on the inferred levels of recent SF and investigate how the mass functions of these subsamples evolve with time. Our information regarding each galaxy's SFH comes from fitting its SED to the nine-band s82-MGC photometry. At the lowest redshifts we consider, $z=0.3$, the SDSS $u$ band samples the rest-frame near-UV, allowing us to constrain the presence of young stars in a similar way as SDSS-I $z \approx 0.1$ studies employing UV data from GALEX (e.g., Salim et al. 2007). The near-IR bands help discriminate between reddening due to dust extinction and the red colors of aging stellar populations (see Paper I).

Figure 8 plots the redshift-dependent distribution of derived SFRs for $\mathrm{S} 82-\mathrm{MGC}$ galaxies with $\log M_{*} / M_{\odot}>11.3 \mathrm{using}$ medians of the SFR posteriors reported by iSEDfit. The distribution of specific star formation rates (sSFRs) is qualitatively similar because of the narrow $M_{*}$ range of our sample but is uniformly low (these are passive galaxies). We therefore focus on the unnormalized SFR, given our interest in low-level, residual SF and the negligible impact such SF has on $M_{*}$ growth for our sample. With the majority of SFR values below $1 M_{\odot} \mathrm{yr}^{-1}$, their accuracy likely depends strongly on the SFH priors we have adopted, which include a (poorly constrained) prescription for bursts. This is acceptable if our goal is to use these SFR estimates as a proxy for examining broad differences in recent SFH across the high-mass population. Other expressions of these differences, such as the birth parameter, $b_{1000}$, or stellar age, yield similar behavior. With this in mind, we divide the SFR distribution into three subsamples. We label galaxies with $\log \mathrm{SFR}<-2.7$ as having "no star formation." Those with $-2.7<\log$ SFR $<-0.5$ are interpreted as having experienced trace amounts of recent SF 

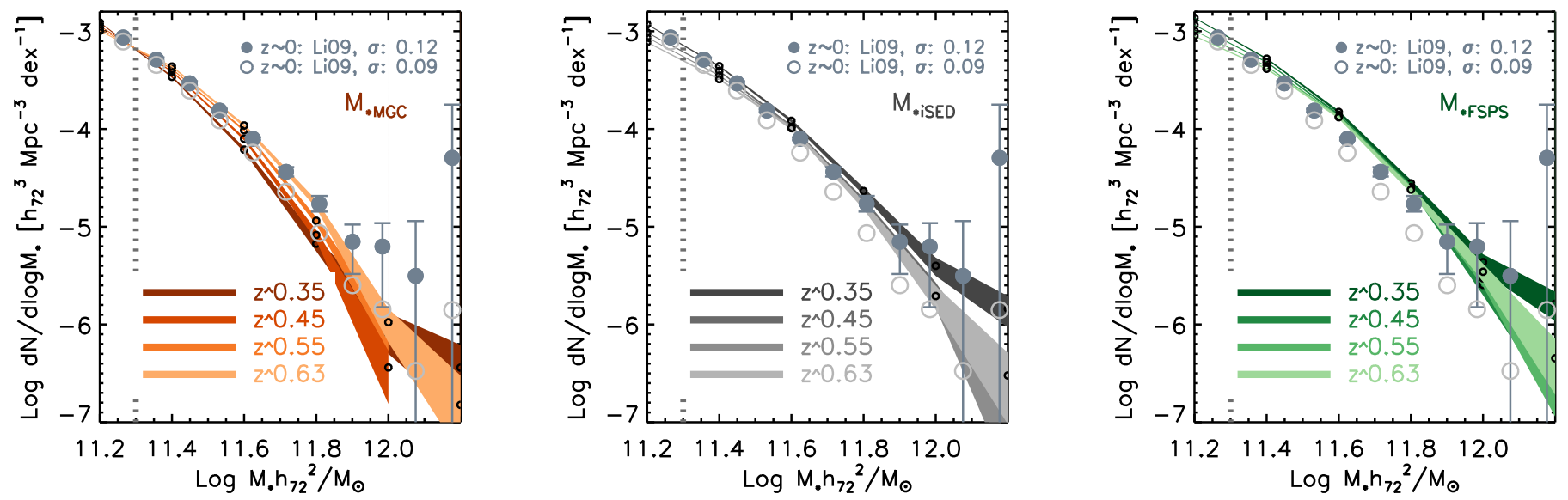

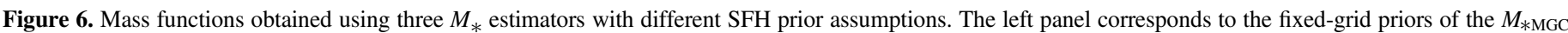

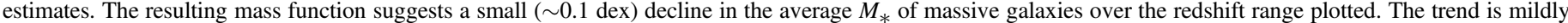

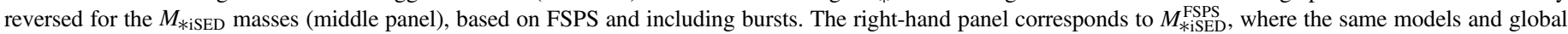
SFH priors have been assumed as in $M_{* \text { *SED }}$, but no bursts are allowed.

and labeled as "minimally" star-forming, while those with $\log$ SFR $>-0.5$ are considered to have ongoing SF.

The evolution of the $\log$ SFR distribution suggests that our classification scheme may have physical meaning. At $z \approx 0.6$, Figure 8 suggests that most high-mass galaxies are quiescent but have some minimal recent SF. As time advances, this population declines, and the majority of our sample falls into the non-starforming category. It is interesting that this evolution suggests an exchange between two modes of behavior, as opposed to a smooth decrease in inferred SFR with time. Meanwhile, a starforming subsample remains present and relatively consistent across the full redshift range.

We can gain further insight by studying how the stellar mass functions of these SFH subsamples evolve with time. The three panels in Figure 9 correspond to the "no SF," "minimal SF," and "ongoing SF" populations. Here we see that the evolutionary signal apparent in Figure 8 is driven by galaxies at the lower-mass end of our sample, that is, with $\log M_{*} / M_{\odot} \lesssim 11.8$. The increase in the no-SF sample coupled with the decline of the minimally star-forming populations at similar masses suggests an exchange, especially given that the total mass function remains essentially fixed. At the highest masses, $\log M_{*} / M_{\odot} \gtrsim 11.8$, most galaxies remain in the minimally star-forming category at all redshifts.

The right-hand panel of Figure 9 reveals the mass function of the star-forming population to be nearly constant with time. Its shape does not follow the total mass function but looks more like a power law. Remarkably, we see that the fraction of galaxies with ongoing SF increases at the highest masses, and, while the statistical uncertainties in our highest-mass bin, $\log M_{*} / M_{\odot}=12.2$, are too large to draw firm conclusions, there is a hint that the majority of galaxies with such extreme $M_{*}$ estimates harbor a degree of ongoing SF at all redshifts.

\section{Discussion}

\subsection{Confidence in Detecting No Evolution}

Even after accounting for $z$-dependent scatter, our assumption-averaged estimate of the high- $M_{*}$ mass function is consistent with no evolution over $0.3<z<0.65$. Here we summarize how different uncertainties affect this conclusion and limit the degree of confidence associated with our claim of a lack of $M_{*}$ growth in the present analysis.
Poisson errors are essentially negligible, especially because any measure of $M_{*}$ growth would average the several mass bins we sample at $\log M_{*} / M_{\odot}>11.3$, while Poisson statistics are independent in each bin. This is not the case for the remaining sample ("cosmic") variance uncertainties, which are highly covariant between mass bins (see Figure 11). Our mock S82MGC catalog suggests a 0.02 dex number density uncertainty for the mass function in the smallest-volume, low- $z$ bin. A number density deviation in one redshift bin at this level could be misinterpreted as a 0.005 dex evolution in the average $M_{*}$. It is unlikely that all four of our redshift bins would suffer systematically increasing sample variance offsets, thereby conspiring to hide underlying $M_{*}$ growth. Still, a conservative estimate for the amount of $M_{*}$ evolution that could be hidden would be a $2 \sigma$ trend across redshift amounting to 0.01 dex.

We have made significant effort addressing concerns over the use of photometric redshifts, particularly their impact on $M_{*}$ scatter (Section 4.2). Regarding conclusions on global evolution, it is important to emphasize that the spectroscopic completeness of these mass functions reaches $80 \%$ above $\log M_{*} / M_{\odot} \approx 11.6$ (Chabrier IMF), even at the highest redshifts. Systematic losses due to completeness are therefore an unlikely contributor to our overall uncertainties. The more general challenge of estimating the $M_{*}$ scatter could be important, however. In other words, it would be helpful to quantify the error on our error estimates. In our effort to make comparisons with the $z=0$ mass function, we noticed that the difference in assuming a total $M_{*}$ measurement scatter of $\sigma=0.12$ dex versus $\sigma=0.09$ leads to a changing mass function that could be misinterpreted as implying $0.07 \mathrm{dex}$ of $M_{*}$ evolution. However, an $\sim 30 \%$ systematic offset in our estimates of $\sigma$ versus their true values seems unlikely over the well-detected high-mass galaxies in our redshift range. A more reasonable estimate for a potential systematic would be 0.02 dex.

In comparison to those above, the most significant systematic errors we have studied so far are the potentially $z$-dependent biases in $M_{*}$ estimates under different assumptions for SFH (Section 5.5). Of the four different $M_{*}$ we combine in our assumption-averaged mass functions, the fiducial $M_{* \mathrm{MGC}}$ estimates (used alone) would indicate a significantly measured decrease in $M_{*}$ over the redshift range. The $M_{* \text { iSED }}$ estimates employing bursts would indicate a slight growth, while the BC03 $M_{* \mathrm{SEDD}}^{\mathrm{BC03}}$ and Maraston $M_{* \mathrm{iSED}}^{\mathrm{Ma05}}$ (neither with bursts) would give no evolution. Although a bursty SFH might be inconsistent 

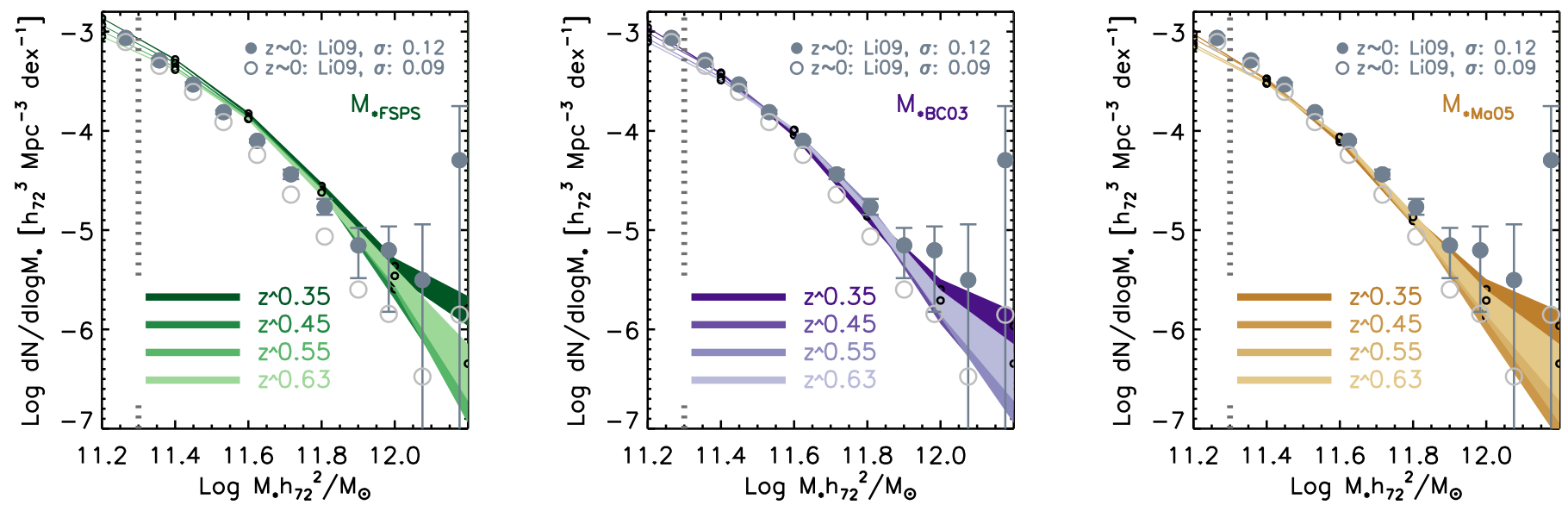

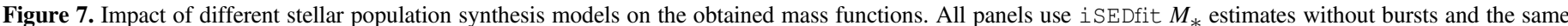

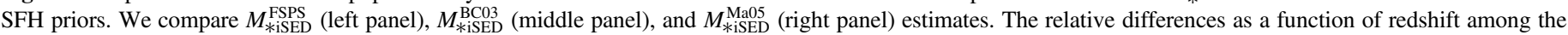
different stellar population synthesis models are subdominant compared to the impact of assuming different priors (Figure 6).

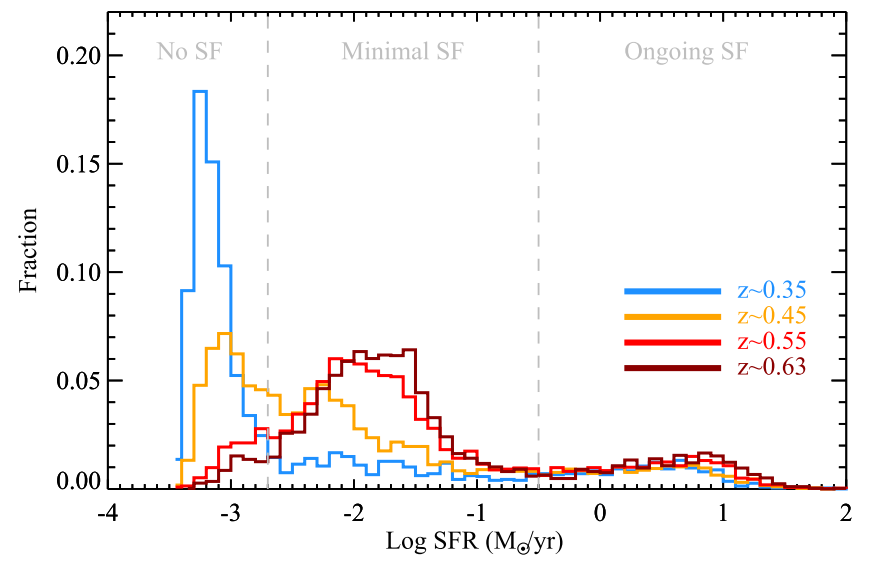

Figure 8. Distribution in SFRs inferred from iSEDfit in different redshift bins for galaxies with $\log M_{*} / M_{\odot}>11.3$. We classify galaxies into three groups, as indicated by the vertical dashed lines. Systems with ongoing SF, present at all redshifts, fall on the right-hand side of the distribution. A population with low, but possibly nonzero, SFRs lies at the center. This mid-SF population decreases with time. On the far left, galaxies with the lowest derived SFRs, consistent with a complete lack of young stars, become increasingly abundant with time and dominate at the lowest redshifts.

with observed $\alpha$-enhanced stellar populations (e.g., Thomas et al. 2005), our current uncertainty in what priors to adopt leads us to combine these $M_{*}$ estimates with equal weight and assess the resulting error on the derived $M_{*}$ evolution to be 0.03 dex.

Combining these systematic error terms in quadrature yields 0.037 dex, suggesting that our results are consistent with $9 \%$ or less evolution in the typical $M_{*}$ of high-mass galaxies over our redshift range.

There is one additional source of potential systematic error that will be addressed in future work and could dominate over the $9 \%$ estimate we quote above, namely, a bias in our estimates of total luminosity. We discuss this uncertainty in more detail below.

\subsection{Biases from Luminosity Estimators}

Stellar mass estimates ultimately rely on measures of the total luminosity of galaxies. Even with $z \approx 0$ SDSS samples, choices in how surface brightness profiles are fit can have dramatic implications for derived $M_{*}$ estimates and resulting stellar mass functions (Bernardi et al. 2013). At the highest masses, discrepancies in $M_{*}$ estimates can reach two orders of magnitude, depending on profile-fitting assumptions (Bernardi et al. 2017; Huang et al. 2017).

Detailed work on nearby galaxies has emphasized the multicomponent nature of galaxy light profiles-spheroidal galaxies often exhibit an outer component that, while low in surface brightness, can contribute significantly to total $M_{*}$ (Huang et al. 2013). There is evidence that the outer components of the most massive central galaxies grow with time even since $z \sim 0.6$ (Vulcani et al. 2014) and that their rising importance accounts for a degree of claimed size evolution (e.g., van der Wel et al. 2014).

Indeed, studies of the evolving mass-size relation put a premium on deep photometry, often from the Hubble Space Telescope, and pay close attention to biases in 2D profile fitting. Unfortunately, the photometric data sets that underlie the galaxy redshift surveys on which number density studies are often based (including this one) are much shallower. Photometry requirements are typically just deep enough to detect galaxies in the sample, not to characterize their low-surface-brightness outskirts. Tal \& van Dokkum (2011) used stacking analyses, for example, to show that SDSS imaging misses $20 \%$ of the total light of luminous red galaxy samples. Shallow imaging depths also motivate the use of rather simple total luminosity estimators, such as the Kron and Hall estimators that underlie our $M_{*}$ estimates in the S82-MGC.

We therefore consider a major limitation of this work to be our inability to quantify the stellar content of the outer components of massive galaxies. Future work exploiting deeper data sets like the Hyper Suprime-Cam Survey may reveal significant growth in these components, which remain below the detection level of the S82-MGC even at the lowest redshifts probed. It is possible that their presence could have a profound effect on conclusions regarding evolution in the total mass function.

\subsection{Comparisons to Other Results}

Figure 10 presents a comparison of the S82-MGC mass functions to both theoretical results (left panel) and recent observational work spanning large volumes (right panel). In both cases, we reproduce from Figure 4 the raw number counts from the assumption-averaged S82-MGC mass function with associated error bars indicated by shaded regions, as well as fits from forward modeling the raw results (thick dotted lines). The forward models account for our estimates of various sources of measurement error. 

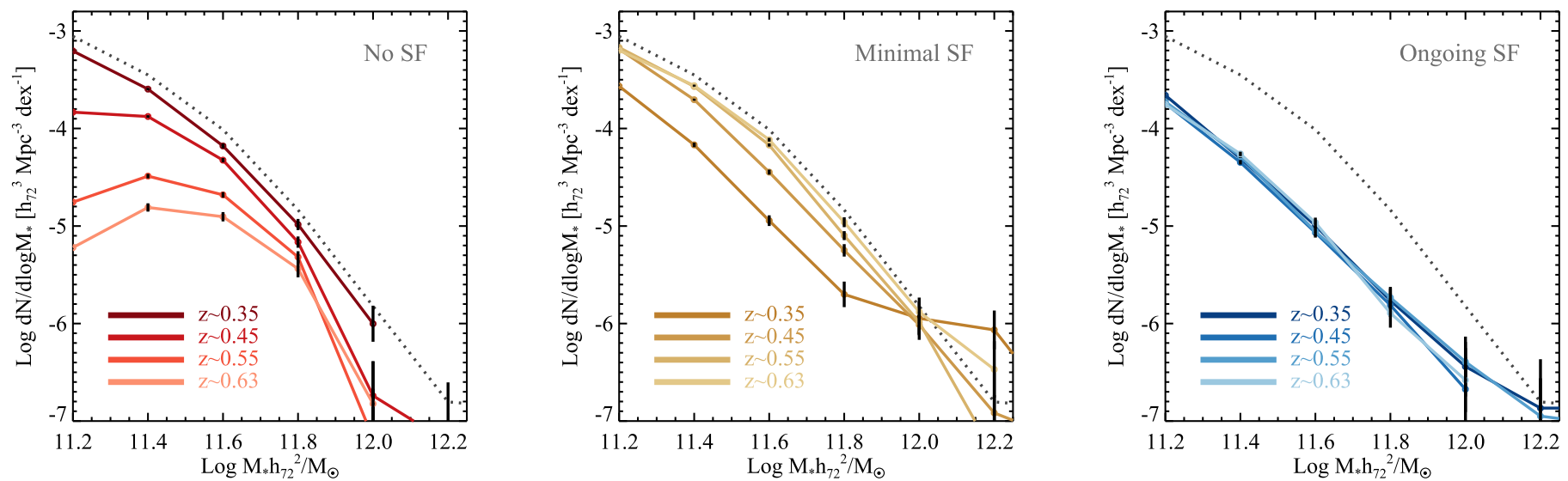

Figure 9. Evolving stellar mass functions of massive galaxies partitioned by the degree of recent SF activity as derived from SED fitting. Each panel corresponds to one of the three populations defined by cuts in the SFR distribution indicated in Figure 8. The rising abundance of completely passive galaxies (left panel), as well as the declining numbers of minimally star-forming galaxies (middle panel), takes place at the lower end of the mass range studied in this work. The highest masses (near $10^{12} M_{\odot}$ ) tend to be dominated by minimally star-forming galaxies at all redshifts. Meanwhile, the mass function of galaxies with residual SF hardly evolves and, interestingly, represents a greater fraction of the total population at the highest masses. In all panels, the total mass function at $z \sim 0.55$ is plotted for comparison. No corrections for scatter are applied to the plotted number densities.
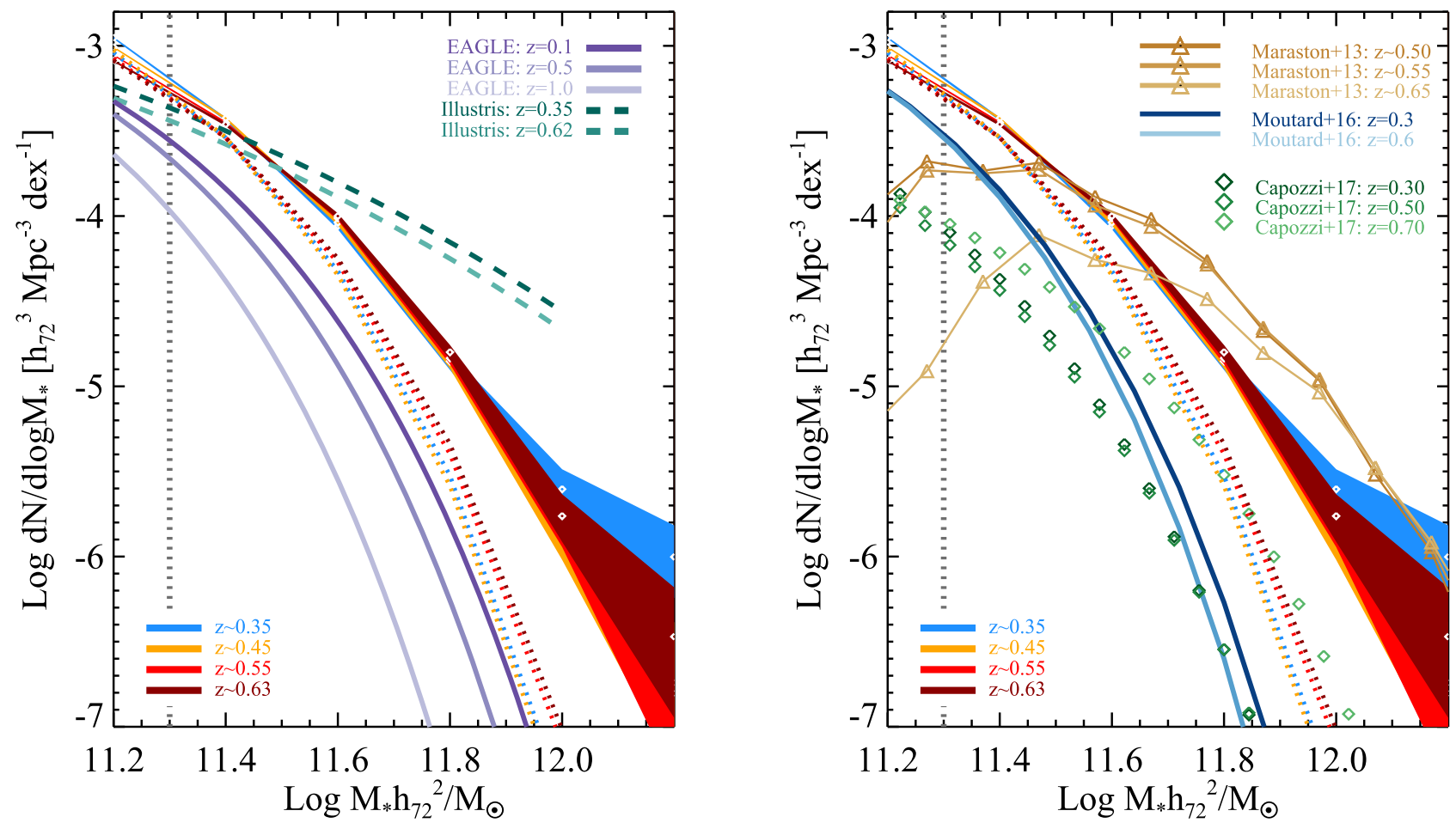

Figure 10. Comparison of mass function fits from cosmological hydrodynamic simulations (left panel) and previous observational results (right panel). Both panels reproduce our assumption-averaged $M_{*}$ mass function results from Figure 4, with the shaded regions indicating the raw number densities and associated error ranges and the thick dotted lines representing the forward-model fitting results after accounting for measurement scatter. In the left panel, the EAGLE mass functions from Furlong et al. (2015) should be compared to the forward-model results, while the Illustris results from Torrey et al. (2017) should be compared to the raw number densities. Simulations predict an $\sim 20 \%$ growth in $M_{*}$ at fixed number density at these masses that is not observed. In the right panel, we reproduce results (Maraston et al. 2013, based on BOSS; Capozzi et al. 2017 based on DES) that include measurement scatter. The VIPERS-based Moutard et al. (2016a) forward models (blue curves) should be compared to our forward-model fits. Global offsets in $M_{*}$ values from different estimators are expected; the sense and strength of claims of internal redshift evolution are of particular interest.

We compare to theoretical results from recent cosmological hydrodynamic simulations (left panel). Stellar mass functions for the EAGLE simulation are taken from fits provided at specific redshifts by Furlong et al. (2015). For a comparison to the Illustris simulation results, we use the mass function fitting formulae provided in Torrey et al. (2017) and evaluate the relation at the midpoint of the lowest and highest redshift bins in the S82-MGC sample. Both simulations predict an $\sim 20 \%-30 \%$ growth in $M_{*}$ at fixed number density at these masses that is not detected in our data. For a direct comparison to Illustris, the raw mass functions may be appropriate, as the Illustris output was tuned to reproduce the evolving galaxy stellar mass function as observed at lower $M_{*}$ (Torrey et al. 2014). These observational results likely included the effects of measurement scatter, which would be expected at $z \gtrsim 0.3$ to be similar to the uncertainties estimated here. We see, however, that the Illustris number densities, while in broad agreement with the $\mathrm{S} 82-\mathrm{MGC}$ at $\log M_{*} / M_{\odot} \approx 11.5$, trace a shallower mass function than what we observe and land an order 
of magnitude too high at $10^{12} M_{\odot}$, although they are in closer agreement with Maraston et al. (2013; see below). At this $M_{*}$, Torrey et al. (2017) warned that Illustris becomes incomplete.

The EAGLE simulation output was tuned to the SDSS-based $z \approx 0.1$ mass function alone, which, being at low redshift, suffers from less measurement error. A direct comparison with EAGLE is therefore more appropriately made with our forward-model fits in which we have attempted to remove the effects of scatter. Here the agreement with our observations in both shape and normalization is better. If applied to the EAGLE results, a constant $M_{*}$ offset of +0.05 dex, well within expectations for mass estimator differences, would bring the low- $z$ mass functions into agreement, and Furlong et al. (2015) speculated that the galaxies in their simulation may be overquenched. Our forward-model results, however, are inconsistent with the smooth redshift evolution predicted by EAGLE (see Section 6.1).

The right-hand panel of Figure 10 compares our results to other observational efforts. The raw number densities derived from the Maraston et al. (2013) analysis of the BOSS sample are plotted with gold open symbols connected by lines. Corrections to $h$ have been applied, but Maraston et al. (2013) did not account for scatter and so should be compared to the raw number counts from the S82MGC. In Paper I, we show that $M_{* \mathrm{MGC}}$ is systematically larger than the Maraston et al. (2013) $M_{*}$ estimates, an offset that increases with $M_{*}$ to $0.1 \mathrm{dex}$ at $\log M_{*} / M_{\odot} \sim 11.8$. The higher Maraston et al. (2013) number densities at fixed $M_{*}$ in Figure 10 may instead result from Eddington bias from larger $M_{*}$ uncertainties (Bundy et al. 2015). Note the effect of CMASS sample incompleteness in the highest redshift bin from Maraston et al. (2013).

The DES photo-z-only Schechter fits from the mass functions in Capozzi et al. (2017) are plotted as green open symbols. These are fits to raw number counts (no scatter correction) and should be compared to our raw number densities (shaded curves). On top of a global $M_{*}$ offset, ${ }^{10}$ the Capozzi et al. (2017) results favor a decreasing mass function with time that would be consistent with a decrease in the typical $M_{*}$ of massive galaxies.

Finally, we plot the forward-model results of Moutard et al. (2016a; blue lines), which are based in part on VIPERS data and should be compared to our forward-model fitting approach (with scatter removed). Acknowledging a global $M_{*}$ offset, the evolutionary signal claimed by Moutard et al. (2016a) appears to have a similar amplitude as the range in forward-modeled mass functions that we derive. Given the uncertainties in our data, we do not interpret this range to be physically meaningful. Separate modeling runs with different random draws of our error distributions yield different relative orientations of our redshiftdependent mass functions. With $22 \mathrm{deg}^{2}$ compared to our $139 \mathrm{deg}^{2}$, the Moutard et al. (2016a) data set may have similar (or greater) uncertainties. The apparent evolution in their mass function fits might therefore arise from differing priors on the mass function shape parameters.

\subsection{Dependence on Star Formation History}

The SFR distributions presented in Figure 8 suggest that massive galaxies can be classified according to the degree of low-level SF that is present. Figure 9 shows that the population

\footnotetext{
${ }^{10}$ The $M_{*}$ estimates in Capozzi et al. (2017) use a Salpeter IMF, which introduces a +0.25 dex offset compared to the Chabrier-based values in this paper. However, the adopted SF priors in Capozzi et al. (2017) were shown by Maraston et al. (2013) to cause a -0.25 dex offset. Since this cancels the offset from the Salpeter IMF compared to our estimates, we plot the Capozzi et al. (2017) results without any corrections applied.
}

with some residual SF stays constant with time, while the abundance of galaxies with minimal SF decreases, apparently resulting in a buildup of systems with no SF at all. These results are based on the optical-near-IR fitting we have performed with iSEDfit and therefore reflect features in broadband SEDs. They are also subject to the adopted priors, which, for example, limit derived SFRs to be greater than $\sim 10^{-3} M_{\odot} \mathrm{yr}^{-1}$, likely resulting in the apparent peak at this value in Figure 8.

Is the apparent decline in the abundance of high-mass galaxies with minimal SF real? If so, it may be a signpost of more recent quenching, past merging episodes with smaller, gas-rich galaxies, or low levels of residual gas cooling and SF that become increasingly rare toward the present day. Alternatively, could the global shift toward near-zero SFRs simply reflect passive evolution of exponentially declining SFHs?

Constraints on SFHs from detailed analysis of massive galaxy spectra present a complementary view (e.g., Thomas et al. 2005, 2010; Tojeiro et al. 2007). For $\log M_{*} / M_{\odot}<11.5$, Choi et al. (2014) stacked $z \sim 0.5$ spectra to argue that more massive quiescent galaxies have older single stellar population (SSP)equivalent ages at all times (for $z \lesssim 1$ ). However, while this agemass trend generally evolves toward older ages with time, the lower-mass galaxy populations age less rapidly. This suggests more complex SFHs, possibly resulting from recent red-sequence arrivals that may also contribute to the "minimal" SFR population we identify here.

Choi et al. (2014) presented exponential SFHs that are meant to globally capture the mass and age trends of their stacked samples. The data are broadly consistent with a short burst ( $\tau=0.1 \mathrm{Gyr}$ ) of SF at $z \sim 1.2$, as well as with longer declining histories $(\tau \sim 2 \mathrm{Gyr})$ initiated at $z=3$. Neither of these global models explain the SFR distributions we see in Figure 8. Short bursts at $z \sim 1$ have completely extinguished by $z \sim 0.5$, and, even if our absolute measure of SFR could be made consistent, the longer SFH models predict $0.2-0.3$ dex of gradual decline in SFR per 0.1-wide redshift bin. Our estimates suggest a much more dramatic cessation. While the exponential models may provide a useful description for the majority of stars in massive galaxies, we conclude that residual low-level SF may still be present in ways that shed light on recent assembly history.

We turn now to the more rare phenomenon of very massive galaxies exhibiting significant levels of SF, with $\mathrm{SFR} \gtrsim 1$ $M_{\odot} \mathrm{yr}^{-1}$. One concern is that our SED-based SFR estimates are biased by a "UV upturn" that is likely a signature of stellar evolution, not a sign of recent SF. Figure 13 in Paper I shows how a related measure of recent $\mathrm{SF}$, the $b_{1000}$ parameter, varies across the optical-near-IR color space of our sample. This plot demonstrates that the majority of our modestly star-forming galaxies have red optical colors. The near-IR photometry is what allows us to distinguish them as (mildly dust-obscured) star formers, not a detection of enhanced UV flux. We also reported that the visual morphologies of these galaxies are predominantly disklike or disturbed.

This star-forming population remains remarkably constant across our redshift range. From $10^{11}$ to $10^{12} M_{\odot}$, roughly $10 \%$ of massive galaxies are in the throes of a noticeable star-forming episode. While unlikely to build significant additional stellar mass, these episodes may again be signs of an active (minor) merging history that in some cases may significantly revive quiescent galaxies (e.g., Kannappan et al. 2009). At $10^{12} M_{\odot}$ and above, galaxies with significant SF appear to be far more common. Assuming that most of these systems are brightest cluster galaxies (BCGs), these results 

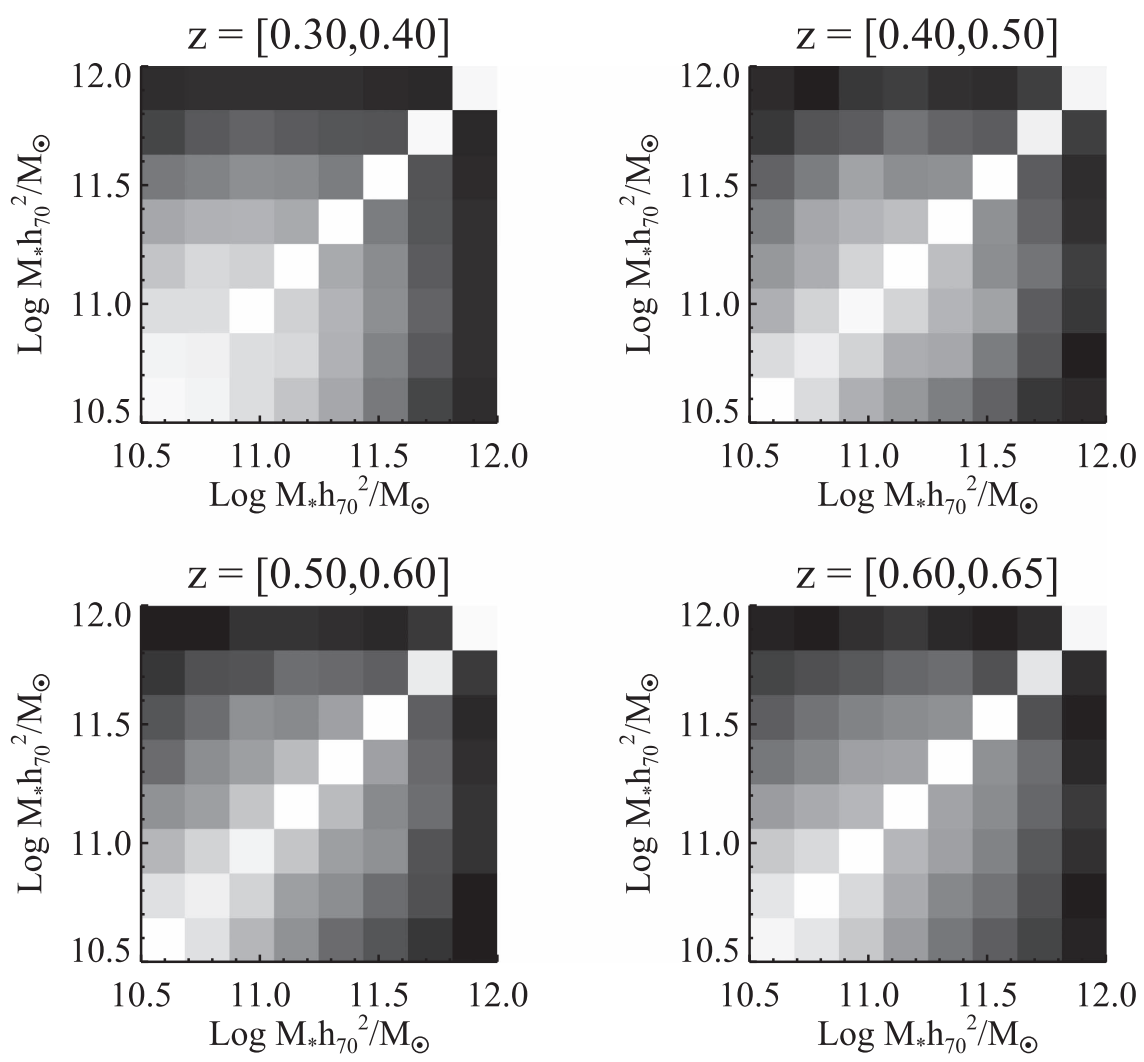

Figure 11. Correlation matrices from the normalized covariance of the $\$ 82-\mathrm{MGC}$ mass functions as determined from gridding the survey footprint into 214 subregions and resampling with replacement.

are consistent with the BCG sample studied by McDonald et al. (2016). They found that $34 \%$ of BCGs at $0.25<z<1.25$ have SFR $>10 M_{\odot} \mathrm{yr}^{-1}$. At $z<0.6$, McDonald et al. (2016) used entropy profiles in the hot intracluster medium (ICM) to argue that cooling in relaxed cool-core clusters provides the dominant source of SFR fuel. The rising fraction of star formers we see in our sample toward higher $M_{*}$ may signal the increasing role of ICM cooling in triggering high- $M_{*}$ SF. Unfortunately, statistical uncertainties limit our ability to study the evolution in the abundance of $M_{*}>10^{12} M_{\odot}$ star formers, and we do not probe beyond $z \sim 0.6$, where McDonald et al. (2016) argued that ICM cooling no longer correlates with BCG SF.

\section{Summary and Conclusions}

We have exploited optical through near-IR matched photometry in the S82-MGC to measure the galaxy stellar mass function in four redshift bins from $z=0.30$ to $z=0.65$. While our $M_{*}$ completeness of $\log M_{*} / M_{\odot}>11.3$ is relatively shallow, our sample spans a large area of $139 \mathrm{deg}^{2}$, delivering exquisite statistical precision on possible evolution at the highest masses.

We pay special attention to sources of random and systematic error and investigate their effects on our derived mass functions through both forward modeling and perturbations to our measurements that result in samples with uniform measurement uncertainties. The two techniques yield consistent results. These techniques also address concerns from the use of photometric redshifts, although our sample has a high degree (80\%) of spectroscopic redshift completeness, even at the highest redshifts we probe.

Our key result is shown in Figure 4. After combining $M_{*}$ estimates that adopt a range of currently uncertain prior assumptions, we find no evolution in the typical $M_{*}$ at fixed number density for massive galaxies in our redshift range. Recent simulations predict growth in $M_{*}$ of $20 \%-30 \%$. Taking into account the errors studied in this work, we can rule out evolution in $M_{*}$ of $9 \%$ or more. Among those considered here, the largest contribution to this uncertainty are biases in $M_{*}$ estimates arising from different SFH priors. However, we speculate that missing light from our adopted total luminosity estimators is of far greater importance and, when accounted for in future work, could strongly impact our conclusions.

Finally, we divide our sample based on the degree of residual low-level SF as determined from our SED fitting. We find a minimally star-forming population that appears to become completely passive over our redshift range. There is an additional less-abundant population with a notable but still low SFR (about $1 M_{\odot} \mathrm{yr}^{-1}$ ) whose mass function hardly evolves. Interestingly, this population becomes more common at the highest masses and may be associated with BCGs in cool-core clusters.

This work was supported by the World Premier International Research Center Initiative (WPI Initiative), MEXT, Japan. This work was supported by Kakenhi Grant-in-Aid for Scientific Research 24740119 from the Japan Society for the Promotion of Science. We thank E. Rykoff and E. Rozo for a generous contribution of redMaPPer photometric redshift estimates. This publication has made use of code written by James R. A. Davenport.

\section{Appendix Covariance Matrices}

The correlation matrices from the bootstrap resampling are plotted in Figure 11 and made available at http://www. massivegalaxies.com. 


\section{ORCID iDs}

Kevin Bundy (i) https://orcid.org/0000-0001-9742-3138

Alexie Leauthaud (1) https://orcid.org/0000-0002-3677-3617

David A. Wake (1) https://orcid.org/0000-0002-6047-1010

\section{References}

Abazajian, K. N., Adelman-McCarthy, J. K., Agüeros, M. A., et al. 2009, ApJS, 182, 543

Annis, J., Soares-Santos, M., Strauss, M. A., et al. 2014, ApJ, 794, 120

Baldry, I. K., Glazebrook, K., \& Driver, S. P. 2008, MNRAS, 388, 945

Behroozi, P. S., Conroy, C., \& Wechsler, R. H. 2010, ApJ, 717, 379

Benson, A. J. 2014, MNRAS, 444, 2599

Benson, A. J., Bower, R. G., Frenk, C. S., et al. 2003, ApJ, 599, 38

Bernardi, M., Meert, A., Sheth, R. K., et al. 2013, MNRAS, 436, 697

Bernardi, M., Meert, A., Sheth, R. K., et al. 2017, MNRAS, 467, 2217

Blanton, M. R., \& Roweis, S. 2007, AJ, 133, 734

Borch, A., Meisenheimer, K., Bell, E. F., et al. 2006, A\&A, 453, 869

Brammer, G. B., Whitaker, K. E., van Dokkum, P. G., et al. 2011, ApJ, 739, 24

Bruzual, G., \& Charlot, S. 2003, MNRAS, 344, 1000

Bundy, K., Ellis, R. S., Conselice, C. J., et al. 2006, ApJ, 651, 120

Bundy, K., Fukugita, M., Ellis, R. S., et al. 2009, ApJ, 697, 1369

Bundy, K., Hogg, D. W., Higgs, T. D., et al. 2012, AJ, 144, 188

Bundy, K., Leauthaud, A., Saito, S., et al. 2015, ApJS, 221, 15

Bundy, K., Scarlata, C., Carollo, C. M., et al. 2010, ApJ, 719, 1969

Capozzi, D., Etherington, J., Thomas, D., et al. 2017, arXiv:1707.09066

Casteels, K. R. V., Conselice, C. J., Bamford, S. P., et al. 2014, MNRAS, 445,1157

Chabrier, G. 2003, PASP, 115, 763

Charlot, S., \& Fall, S. M. 2000, ApJ, 539, 718

Choi, J., Conroy, C., Moustakas, J., et al. 2014, ApJ, 792, 95

Coil, A. L., Blanton, M. R., Burles, S. M., et al. 2011, ApJ, 741, 8

Conroy, C., Gunn, J. E., \& White, M. 2009, ApJ, 699, 486

Davidzon, I., Bolzonella, M., Coupon, J., et al. 2013, A\&A, 558, A23

Dawson, K. S., Schlegel, D. J., Ahn, C. P., et al. 2013, AJ, 145, 10

de Lucia, G., \& Blaizot, J. 2007, MNRAS, 375, 2

Drory, N., Bundy, K., Leauthaud, A., et al. 2009, ApJ, 707, 1595

Eisenstein, D. J., Weinberg, D. H., Agol, E., et al. 2011, AJ, 142, 72

Etherington, J., Thomas, D., Maraston, C., et al. 2017, MNRAS, 466, 228

Furlong, M., Bower, R. G., Theuns, T., et al. 2015, MNRAS, 450, 4486

Garilli, B., Guzzo, L., Scodeggio, M., et al. 2014, A\&A, 562, A23

Gu, M., Conroy, C., \& Behroozi, P. 2016, ApJ, 833, 2

Guo, Q., White, S., Boylan-Kolchin, M., et al. 2011, MNRAS, 413, 101

Guzzo, L., Scodeggio, M., Garilli, B., et al. 2014, A\&A, 566, A108

Hilz, M., Naab, T., \& Ostriker, J. P. 2013, MNRAS, 429, 2924

Hopkins, P. F., Bundy, K., Hernquist, L., Wuyts, S., \& Cox, T. J. 2010, MNRAS, 401, 1099

Huang, S., Ho, L. C., Peng, C. Y., Li, Z.-Y., \& Barth, A. J. 2013, ApJ, 766, 47

Huang, S., Leauthaud, A., Greene, J., et al. 2017, MNRAS, submitted (arXiv:1707.01904)

Ilbert, O., McCracken, H. J., Le Fèvre, O., et al. 2013, A\&A, 556, A55

Ilbert, O., Salvato, M., Le Floc'h, E., et al. 2010, ApJ, 709, 644

Kannappan, S. J., Guie, J. M., \& Baker, A. J. 2009, AJ, 138, 579

Lawrence, A., Warren, S. J., Almaini, O., et al. 2007, MNRAS, 379, 1599
Leauthaud, A., Bundy, K., Saito, S., et al. 2016, MNRAS, 457, 4021

Lee, J., \& Yi, S. K. 2013, ApJ, 766, 38

Li, C., \& White, S. D. M. 2009, MNRAS, 398, 2177

Lidman, C., Iacobuta, G., Bauer, A. E., et al. 2013, MNRAS, 433, 825

López-Sanjuan, C., Le Fèvre, O., Ilbert, O., et al. 2012, A\&A, 548, A7

Lotz, J. M., Jonsson, P., Cox, T. J., et al. 2011, ApJ, 742, 103

Maraston, C. 2005, MNRAS, 362, 799

Maraston, C., Pforr, J., Henriques, B. M., et al. 2013, MNRAS, 435 2764

Marchesini, D., van Dokkum, P. G., Förster Schreiber, N. M., et al. 2009, ApJ, 701, 1765

Matsuoka, Y., \& Kawara, K. 2010, MNRAS, 405, 100

McDonald, M., Stalder, B., Bayliss, M., et al. 2016, ApJ, 817, 86

Moster, B. P., Somerville, R. S., Newman, J. A., \& Rix, H.-W. 2011, ApJ, 731,113

Moustakas, J., Coil, A. L., Aird, J., et al. 2013, ApJ, 767, 50

Moutard, T., Arnouts, S., Ilbert, O., et al. 2016a, A\&A, 590, A102

Moutard, T., Arnouts, S., Ilbert, O., et al. 2016b, A\&A, 590, A103

Mundy, C. J., Conselice, C. J., Duncan, K. J., et al. 2017, MNRAS, 470, 3507

Muzzin, A., Marchesini, D., Stefanon, M., et al. 2013, ApJ, 777, 18

Newman, A. B., Ellis, R. S., Bundy, K., \& Treu, T. 2012, ApJ, 746, 162

Nipoti, C., Treu, T., Leauthaud, A., et al. 2012, MNRAS, 422, 1714

Ownsworth, J. R., Conselice, C. J., Mortlock, A., et al. 2014, MNRAS, 445, 2198

Peng, Y., Lilly, S. J., Kovač, K., et al. 2010, ApJ, 721, 193

Pforr, J., Maraston, C., \& Tonini, C. 2013, MNRAS, 435, 1389

Qu, Y., Helly, J. C., Bower, R. G., et al. 2017, MNRAS, 464, 1659

Reis, R. R. R., Soares-Santos, M., Annis, J., et al. 2012, ApJ, 747, 59

Rozo, E., Rykoff, E. S., Abate, A., et al. 2016, MNRAS, 461, 1431

Rykoff, E. S., Rozo, E., Busha, M. T., et al. 2014, ApJ, 785, 104

Saito, S., Leauthaud, A., Hearin, A. P., et al. 2016, MNRAS, 460, 1457

Salim, S., Rich, R. M., Charlot, S., et al. 2007, ApJS, 173, 267

Stringer, M. J., Benson, A. J., Bundy, K., Ellis, R. S., \& Quetin, E. L. 2009, MNRAS, 393, 1127

Tal, T., \& van Dokkum, P. G. 2011, ApJ, 731, 89

Thomas, D., Maraston, C., Bender, R., \& Mendes de Oliveira, C. 2005, ApJ, 621,673

Thomas, D., Maraston, C., Schawinski, K., Sarzi, M., \& Silk, J. 2010, MNRAS, 404, 1775

Tinker, J. L., Brownstein, J. R., Guo, H., et al. 2017, ApJ, 839, 121

Tojeiro, R., Heavens, A. F., Jimenez, R., \& Panter, B. 2007, MNRAS, 381,1252

Torrey, P., Vogelsberger, M., Genel, S., et al. 2014, MNRAS, 438, 1985

Torrey, P., Wellons, S., Ma, C.-P., Hopkins, P. F., \& Vogelsberger, M. 2017, MNRAS, 467, 4872

van der Wel, A., Franx, M., van Dokkum, P. G., et al. 2014, ApJ, 788, 28

Vikhlinin, A., Kravtsov, A. V., Burenin, R. A., et al. 2009, ApJ, 692, 1060

Vulcani, B., Bundy, K., Lackner, C., et al. 2014, ApJ, 797, 62

Wake, D. A., Nichol, R. C., Eisenstein, D. J., et al. 2006, MNRAS, 372 537

Welker, C., Dubois, Y., Devriendt, J., et al. 2017, MNRAS, 465, 1241

Williams, R. J., Kelson, D. D., Mulchaey, J. S., et al. 2012, ApJL, 749, L12

Wilman, D. J., Fontanot, F., De Lucia, G., Erwin, P., \& Monaco, P. 2013, MNRAS, 433, 2986

Zhao, D., Aragón-Salamanca, A., \& Conselice, C. J. 2015, MNRAS, 453 4444 